\title{
Spectral gradients in central cluster galaxies: further evidence of star formation in cooling flows
}

\author{
N. Cardiel, ${ }^{1}$ J. Gorgas ${ }^{1}$ and A. Aragón-Salamanca ${ }^{2}$ \\ ${ }^{1}$ Departamento de Astrofísica, Facultad de Ciencias Físicas, Universidad Complutense de Madrid, 28040 Madrid, Spain \\ ${ }^{2}$ Institute of Astronomy, Madingley Road, Cambridge CB3 OHA
}

Accepted 1998 March 6. Received 1997 November 27

\begin{abstract}
A B S T R A C T
We have obtained radial gradients in the spectral features of the $\lambda 4000-\AA$ break $\left(\mathrm{D}_{4000}\right)$ and $\mathrm{Mg}_{2}$ for a sample of 11 central cluster galaxies (CCGs): eight in clusters with cooling flows and three in clusters without. After careful removal of the emission lines found within the $\mathrm{D}_{4000}$ and $\mathrm{Mg}_{2}$ bandpasses for some objects, the new data strongly confirm the correlations between line-strength indices and the cooling flow phenomenon found in our earlier study. We find that such correlations depend on the presence and characteristics of emission lines in the inner regions of the CCGs. The nuclear indices are correlated with the mass deposition rate $(\dot{M})$ only when emission lines are found in the central regions of the galaxies. The central $\mathrm{D}_{4000}$ and $\mathrm{Mg}_{2}$ indices in cooling flow galaxies without emission lines are completely consistent with the indices measured in CCGs in clusters without cooling flows. CCGs in cooling flow clusters exhibit a clear sequence in the $\mathrm{D}_{4000}-\mathrm{Mg}_{2}$ plane, with a neat segregation depending on emission-line type and blue morphology. This sequence can be modelled, using stellar population models with a normal initial mass function (IMF), by a recent $(\sim 0.1$ Gyr old) burst of star formation, although model uncertainties do not allow us to completely discard continuous star formation or a series of bursts over the last few Gyr. In CCGs with emission lines, the gradients in the spectral indices are flat or positive inside the emission-line regions, suggesting the presence of young stars. Outside the emission-line regions, and in cooling flow galaxies without emission lines, gradients are negative and consistent with those measured in CCGs in clusters without cooling flows and giant elliptical galaxies. Index gradients measured exclusively in the emission-line region correlate with $\dot{M}$. Using the same population models we have estimated the radial profiles of the mass transformed into new stars. The derived profiles are remarkably parallel to the expected radial behaviour of the mass deposition rate derived from X-ray observations. Moreover, a large fraction ( probably most) of the cooling flow gas accreted into the emission-line region is converted into stars. In the light of these new data, we discuss the evolutionary sequence suggested by McNamara, in which radio-triggered star formation bursts take place several times during the lifetime of the cooling flow. We conclude that this scenario is consistent with the available observations.
\end{abstract}

Key words: stars: formation - cooling flows - galaxies: elliptical and lenticular, cD galaxies: starburst - galaxies: stellar content $-\mathrm{X}$-rays: galaxies.

\section{INTRODUCTION}

The cooling time of the hot intracluster medium is shorter than the Hubble time in the central regions of most galaxy clusters (e.g. Arnaud 1988; Edge, Stewart \& Fabian 1992), which suggests that large amounts of cool material should be settling at the cluster centre. This process, normally known as a cooling flow, may take place at rates of $\dot{M} \sim 100 \mathrm{M}_{\odot} \mathrm{yr}^{-1}$ (Fabian 1994), which implies that $M_{\text {Total }} \sim 10^{12} \mathrm{M}_{\odot}$ of gas would cool and accrete on to the central cluster elliptical galaxies over the lifetime of the clusters. In the idealized picture of a homogeneous cooling flow, all the material will be deposited in the centre of the cluster. However, $\mathrm{X}$-ray data show that the gas is highly inhomogeneous and, very likely, most of the gas must be cooling out at large radii, whilst only a fraction reaches the centre of the flow. In fact, mass deposition profiles are reasonably fitted by $\dot{M}(<r) \propto r$ (Thomas, Fabian \& Nulsen 1987), although some clusters do not follow this trend beyond a certain radius $r \gtrsim 60 \mathrm{kpc}$ (David, Jones \& Forman 1994; 
Irwin \& Sarazin 1995). For comprehensive reviews on cooling flows see Fabian, Nulsen \& Canizares (1984, 1991), Sarazin (1986) and Fabian (1994).

A question of open debate is the nature of the final repository of the cool gas. The discovery of significant excess absorption in the soft X-ray spectra of some galaxy clusters containing cooling flows (White et al. 1991; Johnstone et al. 1992; Miyaji et al. 1993; Allen et al. 1993, 1995; Fabian et al. 1994a; Irwin \& Sarazin 1995; Allen \& Fabian 1997a,b) requires $\sim 10^{11}-10^{12} \mathrm{M}_{\odot}$ of absorbing cold gas, probably in small, pressure-confined, magnetized clouds (Daines, Fabian \& Thomas 1994). This value is comparable to $M_{\text {Total }}$, the mass deposition rate integrated over the age of the clusters. Fabian, Johnstone \& Daines (1994b) have postulated that dust can form in the cold clouds embedded in cooling flows, which would explain why the clouds are almost undetectable outside the X-ray wavelength range. However, there is a great uncertainty concerning the nature of the cold material producing this absorption (Sarazin 1997, Laor 1997). Voit \& Donahue (1995), from the limits imposed by 21cm and CO observations (McNamara, Bregman \& O'Connell 1990; Antonucci \& Barvainis 1994; McNamara \& Jaffe 1994; O’Dea et al. 1994; O’Dea, Gallimore \& Baum 1995), suggest that it is unlikely that significant amounts of cold gas can remain undetectable. On the other hand, very recently Jaffe \& Bremer (1997), using $K$-band spectroscopy, have found that the inner few kpc of central cluster galaxies (CCGs) in cooling flows exhibit strong emission in the $\mathrm{H}_{2}(1-0) \mathrm{S}(1)$ line, which is not seen in a comparable sample of noncooling flow galaxies. These authors suggest that it is likely that a large mass of additional molecular material covers the cooling flow as a whole, producing the soft X-ray absorption.

Since it seems clear that at least a fraction of the cool gas must reach the inner regions of the CCGs, it is reasonable to think that part of this gas could accumulate as molecular clouds which, presumably, would form stars. Unusual diffuse blue light and colours (Sarazin \& O'Connell 1983; Bertola et al. 1986; Johnstone, Fabian \& Nulsen 1987, hereafter JFN87; Romanishin 1987; Crawford et al. 1989; McNamara \& O'Connell 1989, 1992, hereafter MO89, MO92, 1993; Thuan \& Puschell 1989; Nørgaard-Nielsen, Jørgensen \& Hansen 1990; Allen et al. 1992; Crawford \& Fabian 1993; Crawford et al. 1995; Allen 1995; McNamara 1995; Hansen, Jørgensen \& Nørgaard-Nielsen 1995; Cardiel, Gorgas \& AragónSalamanca 1995, hereafter CGA95; Melnik, Gopal-Krishna \& Terlevich 1997), strong low-ionization optical line emission (Baum 1992; Donahue \& Voit 1997, and references therein), and powerful radio sources (see Jaffe 1992 and Burns et al. 1997 for reviews) are usually associated with the inner few kpc of central dominant galaxies in clusters with clear X-ray signatures of cooling flows. Although with a large scatter, the strengths of the optical anomalies are correlated with mass deposition rates (JFN87; MO89; MO92; Heckman et al. 1989, hereafter HBvBM; CGA95; McNamara 1997). In most cases star formation has been invoked to reproduce the excess blue light in the continuum, with typical derived star formation rates ranging from a few to some tens of solar masses per year. It is important to stress that these values normally only account for $\lesssim 10$ per cent of the total X-ray-derived mass deposition rates.

McNamara \& O'Connell (1993) found that the blue colours observed in A 1795 and A 2597 are located in patches that are coincident with the radio lobes of the central galaxies, which suggests a connection between the radio source and the star formation (or, at least, the source of the blue light). Although power-law spectra could also be an alternative explanation for the origin of the excess blue light (e.g. Crawford \& Fabian 1993), the absence of polarization in $U$-band observations of the blue lobes in
A 1795 (McNamara et al. 1996b) makes this scenario unlikely. In addition, observations of this object with the Hubble Space Telescope (McNamara et al. 1996a; Pinkney et al. 1996) and the Ultraviolet Imaging Telescope (Smith et al. 1997) strongly support the idea that the origin of the blue lobes could be an accreted population formed from the cluster cooling flow or from episodes of star formation induced by accretion of stripped material from several gaseous dwarf galaxies.

In this work we address the possible connection between star formation and the cooling flow phenomenon through the study of the stellar populations in CCGs. For this purpose we analyse linestrength gradients in $\mathrm{Mg}_{2}$ (Faber et al. 1985) and the $\lambda 4000-\AA$ break $\left(\mathrm{D}_{4000}\right.$, definition in Bruzual 1983) in a sample of 11 brightest cluster galaxies. This paper is an extension of a previous work presented in CGA95 after the inclusion of extensive new spectroscopic data.

The galaxy sample, reduction of the data and error analysis are presented in Sections 2 and 3. The nuclear line strength and gradients are described in Section 4. The discussion of these results is presented in Section 5, and our conclusions are summarized in Section 6. $H_{0}=50 \mathrm{~km} \mathrm{~s}^{-1} \mathrm{Mpc}^{-1}$ has been assumed throughout the paper.

\section{GALAXY SAMPLE AND OBSERVATIONS}

We have obtained long-slit spectra of CCGs in two runs with different telescopes. Since the aim of the observations was to obtain reliable line-strength gradients, they were performed on dark nights. The first run (1994 August) was carried out with the 3.5-m telescope at the German-Spanish Astronomical Observatory at Calar Alto (Almería, Spain). We used the Cassegrain Twin Spectrograph with a blue-coated TEK CCD in the red channel. In the second run (1995 December) we observed with the 4.2-m WHT at the Roque de los Muchachos Observatory (La Palma, Spain), using the blue arm of ISIS with a blue-coated TEK CCD. A summary of the observations, including relevant instrumental parameters, is given in Table 1.

The sample of CCGs was chosen to cover a wide range of mass deposition rates and complements our previous data (CGA95). It includes eight galaxies in clusters with cooling flows and three galaxies in clusters without cooling flows. A description of the sample, including exposure times and position angles of the spectrograph slit, is listed in Table 2. We have re-observed the central galaxy of A 644, because previous measurements (CGA95) were not clearly understood. We also observed $\mathrm{G}-\mathrm{K}$ giant stars to guarantee that our $\mathrm{Mg}_{2}$ indices are in the appropriate photometric system (see below).

Table 1. Observational configuration.

\begin{tabular}{lcc}
\hline & Run 1 & Run 2 \\
\hline Dates & $9-10$ Aug 1994 & $17-19$ Dec 1995 \\
Telescope & CAHA 3.5-m & WHT 4.2-m \\
Spectrograph & CTS & ISIS blue arm \\
Detector & CCD TEK 12 & CCD TEK 1 \\
Dispersion & $3.46 \AA$ pixel & \\
Wavelength Range & $3700-7240 \AA$ & $2.90 \AA$ pixel $^{-1}$ \\
Spectral Resolution & $8.1 \AA$ (FWHM) & $3740-6700 \AA$ \\
Slit Width & 2.1 arcsec & $12.3 \AA(\mathrm{FWHM})$ \\
Spatial Scale & $0.9 \operatorname{arcsec~pixel~}^{-1}$ & $2.0 \operatorname{arcsec}^{-1}$ \\
\hline
\end{tabular}


Table 2. Galaxy sample.

\begin{tabular}{|c|c|c|c|c|c|c|c|}
\hline Cluster & Galaxy & $\begin{array}{l}\text { R.A. } \\
\text { (1950) }\end{array}$ & $\begin{array}{l}\text { Decl. } \\
(1950)\end{array}$ & $z$ & Run & $\begin{array}{l}\text { Exposure } \\
\text { (s) }\end{array}$ & $\begin{array}{l}\text { P.A. } \\
\left({ }^{\circ}\right)\end{array}$ \\
\hline A 85 & $\ldots$ & 003918.7 & -093441 & 0.0556 & 2 & 7000 & 145 \\
\hline A 478 & $\ldots$ & 041040.7 & +102020 & 0.0859 & 2 & 14000 & 55 \\
\hline A 496 & $\ldots$ & 043118.7 & -132156 & 0.0330 & 2 & 9000 & 175 \\
\hline A 644 & $\ldots$ & 081459.0 & -072123 & 0.0707 & 2 & 9000 & 16 \\
\hline Hydra A & $\ldots$ & 091541.2 & -115305 & 0.0544 & 2 & 8575 & 170 \\
\hline A 779 & N 2832 & 091644.0 & +335740 & 0.0229 & 2 & 7500 & 160 \\
\hline A 1377 & $\ldots$ & 114441.6 & +560028 & 0.0514 & 2 & 1800 & 60 \\
\hline A 2142 & $\ldots$ & 155616.0 & +272233 & 0.0907 & 1 & 15000 & 122 \\
\hline A 2255 & $\ldots$ & 171209.7 & +640706 & 0.0734 & 1 & 12700 & 45 \\
\hline A 2597 & $\ldots$ & 232243.7 & -122400 & 0.0826 & 2 & 3000 & 140 \\
\hline A 2626 & $\ldots$ & 233359.5 & +205208 & 0.0552 & 1 & 12000 & 42 \\
\hline
\end{tabular}

\section{DATA REDUCTION AND ERROR ANALYSIS}

Reduction of the data was carried out using our own reduction package $\mathrm{R}_{\mathrm{E}} \mathrm{D}_{\mathrm{m}}^{\mathrm{uc}} \mathrm{E}$ (Cardiel \& Gorgas, in preparation ${ }^{1}$ ). We followed a standard reduction for long-slit spectroscopic data: bias and dark subtraction, cosmic ray cleaning, flat-fielding, wavelength calibration, C-distortion correction, sky subtraction, extinction corrections, centring and binning of the spectra. The process is similar to that explained in CGA95, and its description is not repeated here. However, we present a summary of the error sources and the procedure followed for their estimation.

\subsection{Random errors}

We describe below the main sources of random errors. Except for the inner parts of the galaxies with long exposure times, these are strongly dominated by photon statistics and read-out noise. The contribution of additional sources of random error is quite negligible.

(i) Photon statistics and read-out noise. These have been computed with the help of a new set of analytic formulae derived by Cardiel et al. (1998). Error images, which are created at an early stage of the reduction procedure, are processed in parallel with data images. The basic arithmetic manipulations performed on the data frames are translated into the error frames following the usual error propagation laws. The errors obtained using this method are in excellent agreement with numerical simulations (see Cardiel et al. 1998 for details). In the outer parts of the galaxies the spectra were spatially binned to guarantee maximum random errors $\Delta \mathrm{D}_{4000} \lesssim 0.15$ and $\Delta \mathrm{Mg}_{2} \lesssim 0.025$ mag.

(ii) Flux calibration. We observed four spectrophotometric standards (Massey et al. 1988; Oke 1990) in each run. The derived calibration curves of each run were averaged and we estimated the uncertainty in flux calibration as the rms scatter among the indices measured with different curves $\left(\Delta \mathrm{D}_{4000} \simeq 0.03, \Delta \mathrm{Mg}_{2} \simeq 0.004\right.$ mag for run 1 , and $\Delta \mathrm{D}_{4000} \simeq 0.03, \Delta \mathrm{Mg}_{2}<0.001$ mag for run 2).

(iii) wavelength calibration. Spectra were transformed to a linear wavelength scale by using $\sim 35$ arc lines fitted by fifthorder polynomials, with typical rms $\simeq 0.3 \AA$ (run 1 ) and $\simeq 0.5 \AA$ (run 2). These scatters are completely consistent with those obtained by cross-correlating the central spectra of the single exposures of each galaxy. These uncertainties translate into $\Delta \mathrm{D}_{4000}<0.01$ and $\Delta \mathrm{Mg}_{2}<0.001 \mathrm{mag}$ for both runs.

\footnotetext{
${ }^{1}$ See also http://www.ucm.es/OTROS/Astrof/reduceme/reduceme.html
}

(iv) Radial velocities. Radial velocities were computed by cross-correlating the central spectra of the final co-added image of each galaxy with different templates (usually $\sim$ four broadened spectra of bright $\mathrm{G}-\mathrm{K}$ giant stars). The different measurements were obtained with a typical rms $\simeq 10 \mathrm{~km} \mathrm{~s}^{-1}$ (run 1) and $\mathrm{rms} \simeq 20 \mathrm{~km} \mathrm{~s}^{-1}$ (run 2), which has a negligible effect in the measurement of the indices $\left(\Delta \mathrm{D}_{4000}<0.01\right.$ and $\Delta \mathrm{Mg}_{2}<0.001$ mag). For comparison, an error of $200 \mathrm{~km} \mathrm{~s}^{-1}$ turns into $\Delta \mathrm{D}_{4000} \simeq 0.01$ and $\Delta \mathrm{Mg}_{2} \simeq 0.004$ mag for both runs.

\subsection{Systematic errors}

Systematic errors in the measurement of spectral indices arise mainly from the effects of spectral resolution (and velocity dispersion), flux calibration, sky subtraction and contamination by nebular emission, among others. Unfortunately, systematic errors do not allow the same straightforward treatment used for the random errors.

(i) Spectral resolution and velocity dispersion. $\mathrm{The}^{\mathrm{Mg}} \mathrm{g}_{2}$ index, which is defined using relatively broad bandpasses, is quite independent of spectral resolution and velocity dispersion corrections (e.g. Gorgas, Efstathiou \& Aragón-Salamanca 1990; González 1993; Carollo, Danziger \& Buson 1993). The $\mathrm{D}_{4000}$, due to its large wavelength coverage, is completely insensitive to these effects. Thus, these particular indices do not require any spectral resolution correction.

(ii) Flux calibration. In order to guarantee that our $\mathrm{Mg}_{2}$ measurements are in a consistent photometric system, we observed a sample of 39 (run 1) and 13 (run 2) template stars (typically G-K giant stars) from the Lick library (Gorgas et al. 1993; Worthey et al. 1994). We compared our $\mathrm{Mg}_{2}$ indices with those of the Lick group in Fig. 1. We found a systematic offset of $0.021 \mathrm{mag}$ (with an rms scatter of $0.010 \mathrm{mag}$ ). This value is almost the same when analysing the data for both runs separately: $0.020(\mathrm{rms}=0.008)$ and 0.021 $(\mathrm{rms}=0.021) \mathrm{mag}$ for runs 1 and 2 , respectively. This offset is similar to that reported in CGA95 (0.014 mag), and it is also consistent with those determined by other authors: e.g. $0.017 \mathrm{mag}$ (Davies, Sadler \& Peletier 1993; González 1993), 0.010 mag (Carollo \& Danziger 1994). This systematic offset is due to the fact that the Lick data were flux calibrated using a tungsten lamp as a relative calibrator of the instrumental spectral response and, thus, they are not in a true photometric system. However, we have transformed our $\mathrm{Mg}_{2}$ indices to the Lick system to perform comparisons with $\mathrm{Mg}_{2}$ published in the literature. 


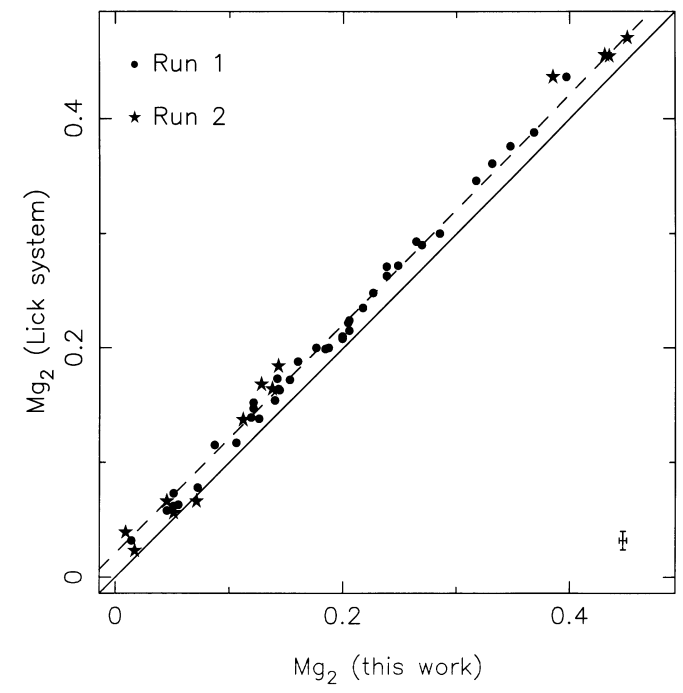

Figure 1. Comparison of our $\mathrm{Mg}_{2}$ measurements with those measured by the Lick group in samples of 39 (run 1) and 13 (run 2) stars from the Lick library. The error bars in the lower right corner indicate the typical random error. There is a systematic offset of $0.021 \mathrm{mag}$ (dashed line) with an rms scatter of $0.010 \mathrm{mag}$. The offsets found by analysing each run independently exhibit an excellent agreement: $0.020, \mathrm{rms}=0.008$ and $0.021, \mathrm{rms}=0.021 \mathrm{mag}$ for run 1 and 2 , respectively.

Unfortunately, the Lick library does not cover the spectral region corresponding to $\mathrm{D}_{4000}$. To check the reliability of the photometric system employed in the determination of this spectral feature we have compared the measurements of four stars in common with CGA95. The scarcity of this sample only allows us to conclude that the $\mathrm{D}_{4000}$ indices measured here and those presented in CGA95 are, within errors, in the same photometric system (offset $=0.17$, $\mathrm{rms}=0.27$ ).

(iii) Sky subtraction. Since galaxy light levels are usually only a few per cent of the sky signal in the outer parts of the galaxies, this process constitutes one of the most important potential sources of systematic errors when measuring line-strength gradients. This problem was treated in CGA95, and we refer the interested reader to that paper. Although there is not a simple recipe to detect a systematic effect in the sky subtraction, anomalous deviations of the line-strength indices in the outer parts of the galaxies are indicative of its presence. We have corrected from the contribution of the galaxy light in the regions where the sky levels were measured by fitting de Vaucouleurs laws to the galaxy profiles. When necessary, we have subtracted from the sky spectra a scaled and averaged galaxy spectrum. Even in the outermost regions of the galaxies the effect of such corrections on the measured indices is nevertheless quite small ( $\sim 10-20$ per cent of the random error).

(iv) Contamination by nebular emission. Emission lines present in the inner parts of some CCGs (e.g. [Ne III] $\lambda 3869$, [S II] $\lambda \lambda 4069,4076, \mathrm{H} \delta,\left[\mathrm{O}\right.$ III] $\left.\left.\lambda 4959, \mathrm{~N}_{\mathrm{I}}\right] \lambda 5199\right)$ must be removed from the bandpasses employed in the measurement of $\mathrm{Mg}_{2}$ and $\mathrm{D}_{4000}$. For this purpose we have followed an interpolation procedure based on a simultaneous fitting of a scaled template spectrum, a low-order polynomial and a Gaussian, in the region close to the emission lines (see Fig. 2). The pixels affected by the presence of emission were carefully replaced by the result of that fit (i.e. polynomial+template). In some objects these corrections are not negligible: e.g. A 2597: $\delta \mathrm{D}_{4000}=0.06$, $\delta \mathrm{Mg}_{2}=0.100 ; \mathrm{A} 478: \delta \mathrm{D}_{4000}=0.02, \delta \mathrm{Mg}_{2}=0.040$; Hydra A:

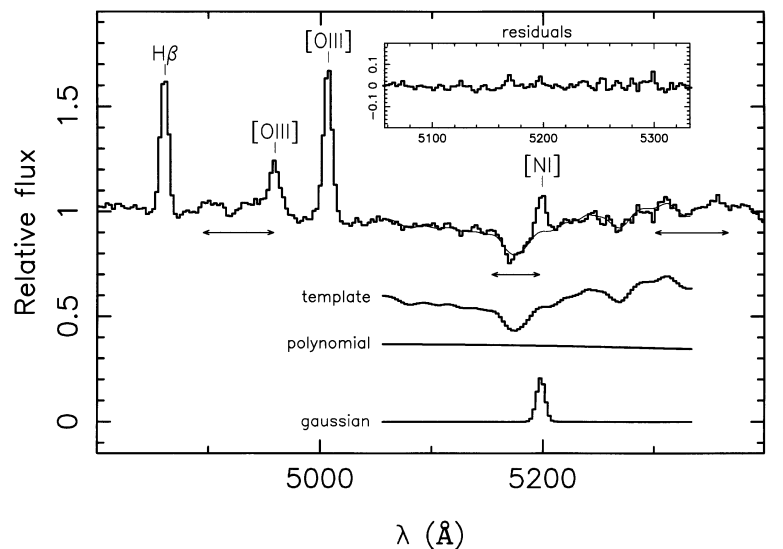

Figure 2. Nuclear spectrum of the central galaxy of the cluster Hydra A with typical emission lines. The arrows indicate the location of the $\mathrm{Mg}_{2}$ bandpasses. A simultaneous fit of a scaled template spectrum, a secondorder polynomial and a Gaussian reproduces the local galaxy spectrum around the central bandpass. The final fit (polynomial+template) is shown as the thin line overplotted on the galaxy spectrum. The rms scatter of the residuals (shown in the inner small box) is 0.017 , absolutely consistent with the mean random error in this spectral range (0.016). The pixels affected by the $[\mathrm{NI}] \lambda 5199$ emission are replaced by this combined fit. The [O III] $\lambda 4959$ emission in the blue bandpass is analogously removed. Common emission lines interpolated in the $\mathrm{D}_{4000}$ bandpasses are [Ne III] $\lambda 3869$, [S II] $\lambda \lambda 4069$, 4076 and $\mathrm{H} \delta$. This procedure is repeated in each spectrum with emission lines.

$\delta \mathrm{D}_{4000}=0.01, \quad \delta \mathrm{Mg}_{2}=0.010 \quad\left(\delta\right.$ Index $=$ Index $_{\mathrm{nel}}-$ Index $_{\mathrm{el}}$, where Index $x_{\mathrm{el}}$ is the index measured without removing the emission lines, and Index $x_{\text {nel }}$ the index obtained when the emission has been interpolated). This procedure has been applied to the whole sample of galaxies with emission lines in their central regions.

\section{RESULTS}

\subsection{Measurement of the central indices}

Following the same procedure as in CGA95, and in order to avoid aperture effects, we have obtained central $\mathrm{D}_{4000}$ and $\mathrm{Mg}_{2}$ indices employing a standard metric aperture size corresponding to 4 arcsec projected at the distance of the Coma cluster $(\simeq 2.6 \mathrm{kpc})$. The central indices presented in CGA95 were not corrected from emission lines. In order to get a fully consistent list of central indices, we have revised the spectra of that sample which exhibited emission lines. We have found that the effect is non-negligible in two objects, namely A 1795 (with $\delta \mathrm{D}_{4000}=0.02$ and $\delta \mathrm{Mg}_{2}=0.026 \mathrm{mag}$ ) and A 2199 (with $\delta \mathrm{D}_{4000}=0.01$ and $\delta \mathrm{Mg}_{2}=0.009 \mathrm{mag}$ ). In addition, we have transformed all the $\mathrm{Mg}_{2}$ measurements to the Lick system by applying the derived systematic offsets. A full list with all the available central measurements and associated random errors, including the revised data from CGA95, is given in Table 3.

\subsection{Line-strength gradients}

We have measured $\mathrm{D}_{4000}$ and $\mathrm{Mg}_{2}$ gradients for all the galaxies of the sample. In order to obtain symmetric brightness profiles at both sides of the galaxy centre, the spectra were shifted by a fraction of a pixel in the spatial direction. This process was not carried out with the central galaxy of A 2626 since in this object a secondary nucleus 
Table 3. Central $D_{4000}$ and $\mathrm{Mg}_{2}$ indices, and associated random errors $\left(\Delta \mathrm{D}_{4000}\right.$ and $\left.\Delta \mathrm{Mg}_{2}\right)$, for the central galaxies of the clusters named in the first column. The measurements correspond to a fixed metric aperture of $4 \operatorname{arcsec}$ at the distance of the Coma cluster $(\simeq 2.6 \mathrm{kpc}$ ). We have included the sample of CGA95, with revised central measurements for A 1795 and A 2199 (due to emission-line contamination). The $\mathrm{Mg}_{2}$ values have been converted to the Lick system using the systematic offsets derived from the observation of template stars (see text). Mass deposition rate, $\dot{M}$, and cooling radius, $r_{\text {cool }}$, for each cluster are taken from: [1] Peres et al. (1998, PSPC data), [2] Edge et al. (1992), [3] MO92, [4] HBvBM, and [5] Arnaud (1988). When available, the values quoted for these last two parameters are the median, 10 and 90 percentile estimates. Central mass deposition rates (i.e. corresponding to the same metric aperture as that employed in the measurement of the line-strength indices) have been obtained assuming $\dot{M} \propto r$. The $r_{\text {cool }}$ value for A 1126 is the mean cooling radius for the four galaxies with similar mass deposition rates (A 85, A 644, Hydra A and A 2597).

\begin{tabular}{|c|c|c|c|c|c|c|c|c|}
\hline Cluster & $\mathrm{D}_{4000}$ & $\Delta \mathrm{D}_{4000}$ & $\begin{array}{l}\mathrm{Mg}_{2} \\
\text { (mag) }\end{array}$ & $\Delta \mathrm{Mg}_{2}$ & $\begin{array}{c}\dot{M} \\
\left(\mathrm{M}_{\odot} \mathrm{yr}^{-1}\right)\end{array}$ & $\begin{array}{l}r_{\text {cool }} \\
(\mathrm{kpc})\end{array}$ & $\begin{array}{l}\text { central } \dot{M} \\
\left(\mathrm{M}_{\odot} \mathrm{yr}^{-1}\right)\end{array}$ & $\begin{array}{l}\text { source of } \\
\dot{M}, r_{\text {cool }}\end{array}$ \\
\hline A 85 & 2.09 & 0.03 & 0.300 & 0.008 & $198_{-52}^{+53}$ & $146_{-41}^{+41}$ & $1.75_{-0.67}^{+0.68}$ & 1 \\
\hline A 262 & 2.34 & 0.04 & 0.330 & 0.009 & $27{ }_{-3}^{+4}$ & $104{ }_{-10}^{+11}$ & $0.33_{-0.05}^{+0.06}$ & 1 \\
\hline 2A $0335+096$ & 2.08 & 0.04 & 0.293 & 0.009 & $325{ }_{-43}^{+32}$ & $215_{-29}^{+29}$ & $1.95_{-0.37}^{+0.32}$ & 1 \\
\hline A 478 & 1.84 & 0.03 & 0.277 & 0.013 & $616_{-76}^{+63}$ & $204_{-38}^{+27}$ & $3.89_{-0.87}^{+0.65}$ & 1 \\
\hline A 496 & 2.25 & 0.04 & 0.318 & 0.003 & $95_{-12}^{+13}$ & $110_{-15}^{+12}$ & $1.11_{-0.20}^{+0.19}$ & 1 \\
\hline PKS 0745-191 & 1.31 & 0.04 & 0.130 & 0.045 & $1038_{-68}^{+116}$ & $214_{-25}^{+49}$ & $6.26_{-0.84}^{+1.59}$ & 1 \\
\hline A 644 & 2.23 & 0.03 & 0.329 & 0.007 & $189_{-35}^{+106}$ & $141_{-18}^{+62}$ & $1.73_{-0.39}^{+1.23}$ & 1 \\
\hline Hydra A & 1.44 & 0.02 & 0.246 & 0.004 & $264{ }_{-60}^{+81}$ & $162{ }_{-68}^{+56}$ & $2.10_{-1.00}^{+0.97}$ & 1 \\
\hline A 779 & 2.28 & 0.04 & 0.347 & 0.002 & 0 & $\ldots$ & $\ldots$ & 3 \\
\hline A 1126 & 1.82 & 0.03 & 0.294 & 0.009 & 222 & $150{ }_{-50}^{+50}$ & $1.91_{-0.64}^{+0.64}$ & 4 \\
\hline A 1377 & 2.26 & 0.04 & 0.321 & 0.009 & 0 & $\ldots$ & $\ldots$ & 5 \\
\hline A 1795 & 1.63 & 0.03 & 0.249 & 0.010 & $381_{-23}^{+41}$ & $177_{-6}^{+19}$ & $2.78_{-0.19}^{+0.42}$ & 1 \\
\hline A 2124 & 2.16 & 0.04 & 0.293 & 0.008 & 0 & $\ldots$ & $\ldots$ & 5 \\
\hline A 2142 & 2.36 & 0.05 & 0.334 & 0.006 & $350{ }_{-133}^{+66}$ & $1500_{-49}^{+18}$ & $3.01_{-1.51}^{+0.67}$ & 1 \\
\hline A 2199 & 2.10 & 0.05 & 0.322 & 0.007 & $154_{-8}^{+18}$ & $143_{-6}^{+17}$ & $1.39_{-0.09}^{+0.23}$ & 1 \\
\hline A 2255 & 2.42 & 0.03 & 0.306 & 0.017 & 0 & $\ldots$ & $\ldots$ & 2 \\
\hline A 2319 & 2.02 & 0.04 & 0.311 & 0.011 & $20{ }_{-20}^{+61}$ & $53_{-53}^{+59}$ & $0.47_{-0.67}^{+1.58}$ & 1 \\
\hline A 2597 & 1.64 & 0.02 & 0.260 & 0.016 & $271_{-41}^{+41}$ & $152_{-58}^{+67}$ & $2.30_{-0.94}^{+1.07}$ & 1 \\
\hline A 2626 & 2.16 & 0.03 & 0.291 & 0.013 & $36_{-36}^{+16}$ & $119_{-60}^{+60}$ & $0.38{ }_{-0.43}^{+0.26}$ & 5 \\
\hline A 2634 & 2.21 & 0.02 & 0.300 & 0.007 & 0 & $\ldots$ & $\ldots$ & 5 \\
\hline
\end{tabular}

was also observed inside the slit at only $\sim 3.4$ arcsec from the centre of the CCG. In this case a simultaneous fit of two Cauchy functions plus a low-order polynomial allows us to determine the location of the centre of the galaxy and the secondary nucleus, together with the relative light contribution of each component near to the central region of the galaxy. The spectra were spatially binned in the outer parts of the galaxies to guarantee a minimum value of the signal-tonoise ratio.

$\mathrm{D}_{4000}$ and $\mathrm{Mg}_{2}$ gradients are plotted in Figs 3 and 4, respectively, where the galaxies are displayed in order of decreasing mass deposition rate (label in the upper right corner of each panel). Galaxies from CGA95 with reliable gradients are also included in these diagrams. Filled circles and stars correspond to measurements on different sides of the galaxies. Open circles refer to secondary nuclei. The thick horizontal solid lines in the upper left corners of the panels indicate the spatial extent of the emission lines, when present. We define the emission-line region as the area where the emission lines were detected above $1 \sigma$. The cut-off of this region is very sharp, so the actual deffinition of its edge is not critical.

In most cases, indices measured at both sides of the galaxies agree within the errors. Error-weighted least-squares fits to all the points, excluding data within the central 1.5 arcsec (affected by seeing) and secondary nuclei, are shown as thin lines for all the galaxies. In addition, we have computed inner and outer gradients (i.e. inside and outside the emission-line region, respectively) in the galaxies with central emission lines. These inner and outer gradients, plotted with a thick line, have been forced to join at the radius where emission lines end. The results obtained in all these fits, together with their formal errors, are given in Table 4 .

\subsection{Comparison with previous work}

We have carried out a comparison of our central $\mathrm{D}_{4000}$ measurements in CCGs (see Table 3) with the available data in the literature. In Fig. 5 we plot $\mathrm{D}_{4000}$ data from JFN87 and MO89, and $(U-b)_{\text {nuc }}$ from MO92 against our central $\mathrm{D}_{4000}$ indices. There is a clear systematic offset of $0.24(\mathrm{rms}=0.04)$ in $\mathrm{D}_{4000}$ between the indices of JFN87 and this work. Although error bars are not available for the JFN87 data, assuming a typical random error of $\Delta D_{4000} \sim 0.04$, the derived offset is probably inconsistent with the intrinsic errors and must be considered as a systematic effect. In contrast, the $\mathrm{D}_{4000}$ values from MO89 are consistent with our measurements since the computed offset is $0.06 \pm 0.08$. The nuclear $(U-b)$ colours from MO92 display a very good correlation with the $\mathrm{D}_{4000}$ ones. 


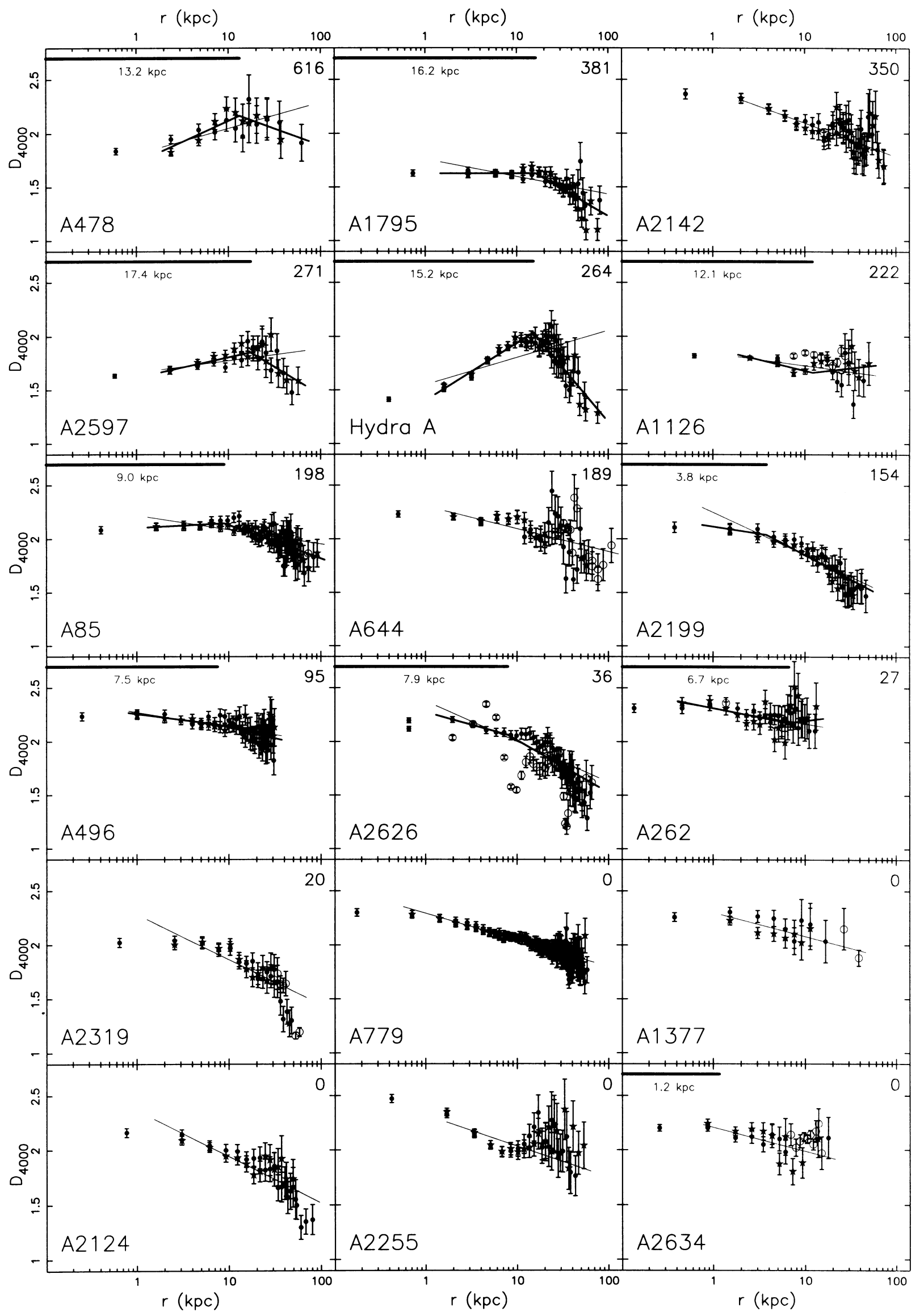

Figure 3. $\mathrm{D}_{4000}$ gradients. See explanation in Section 4.2. 


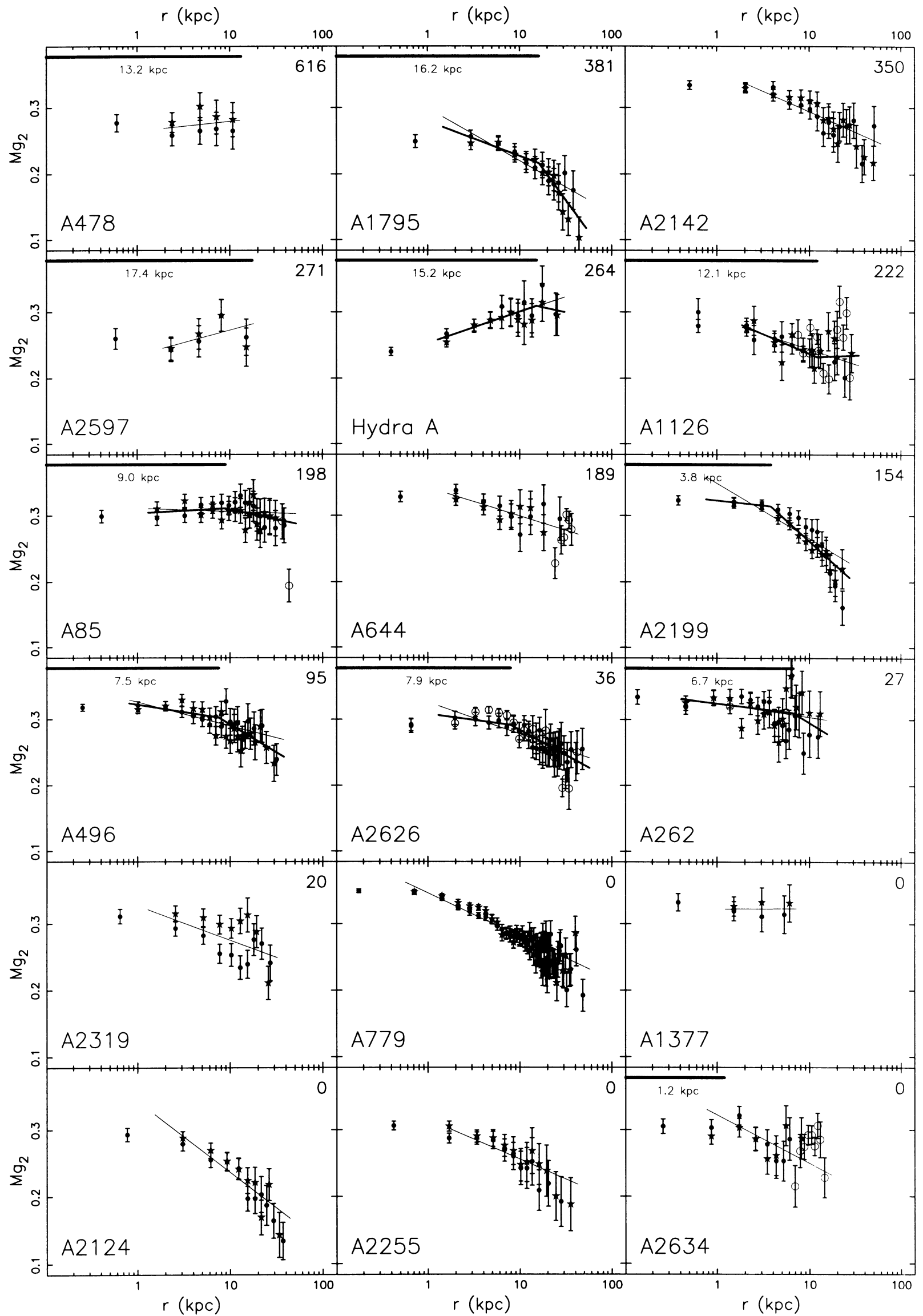

Figure 4. $\mathrm{Mg}_{2}$ gradients. See explanation in Section 4.2. 
Table 4. $\mathrm{D}_{4000}$ and $\mathrm{Mg}_{2}$ gradients $\left(\mathrm{dD}_{4000} / \mathrm{d} \log r, \mathrm{dMg}_{2} / \mathrm{d} \log r\right.$ ), corresponding to the error-weighted least-squares fits shown in Figs 3 and 4 . Total gradients refer to fits computed by employing all the data excluding measurements within 1.5 arcsec and secondary nuclei. Inner and outer gradients correspond to fits inside and outside the emission-line region, when present. Formal errors of the fits are given next to each gradient.

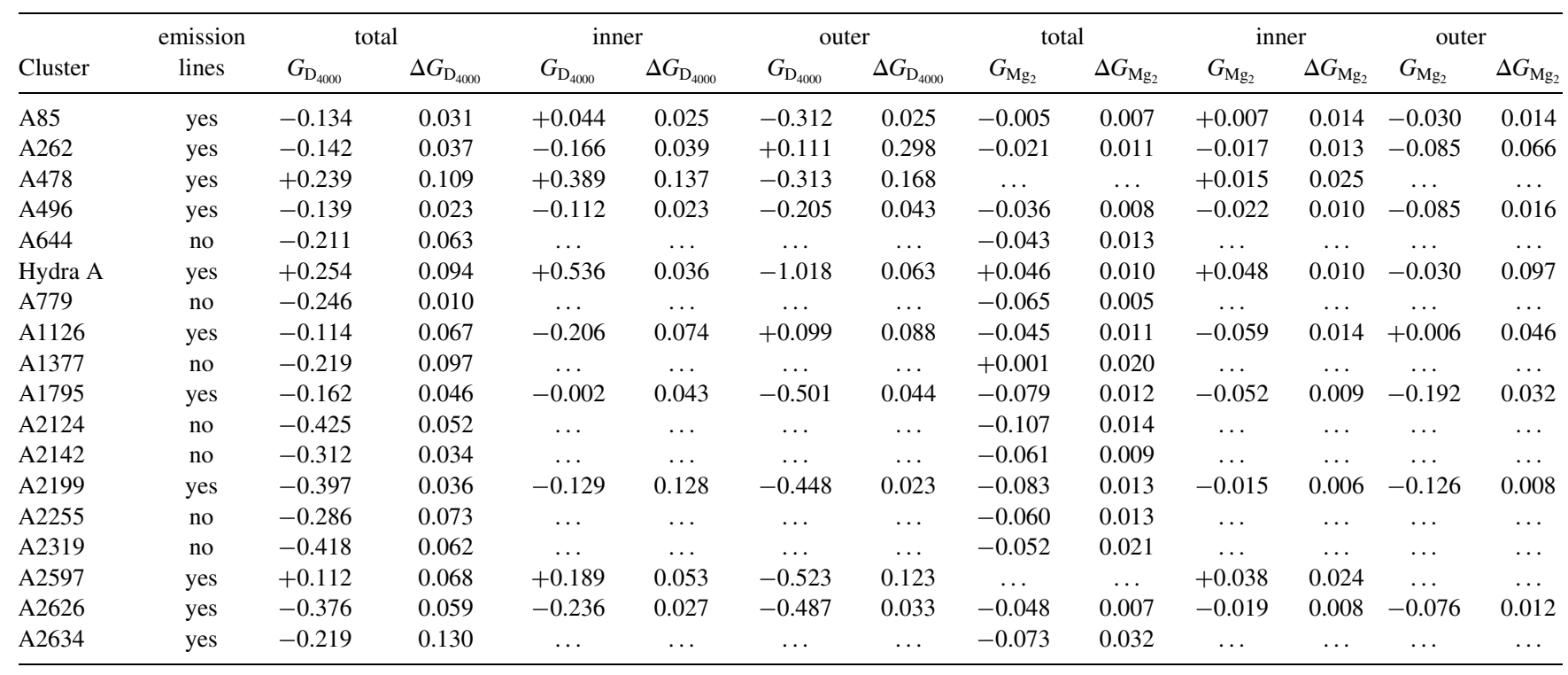

A similar correlation was previously presented by MO89. This correlation can be easily understood since the $(U-b)$ colours measure essentially the same spectral break as $\mathrm{D}_{4000}$. The dashed line in Fig. (5b) is the least-squares fit to the data: $(U-b)_{\text {nuc }}=-1.03( \pm 0.14)+0.81( \pm 0.07) \mathrm{D}_{4000}$. This linear relation will be employed later in this paper to estimate central $\mathrm{D}_{4000}$ from $(U-b)_{\text {nuc }}$ colours.

Line-strength gradients of $\mathrm{Mg}$ in brightest cluster galaxies have been presented by Fisher, Franx \& Illingworth (1995, hereafter FFI95). Our full sample includes four galaxies in common with theirs, namely N 2832 (A 779), N 6166 (A 2199), N 7720 (A 2634; note that this object was mislabelled as N 7728 in CGA95) and the central cluster galaxy of A 496. In order to compare their measurements of $\mathrm{Mgb}$ with ours of $\mathrm{Mg}_{2}$ we have transformed $\mathrm{Mgb}$ into $\mathrm{Mg}_{2}$. The atomic Mgb index is very well correlated with the molecular $\mathrm{Mg}_{2}$ index in early-type systems: González \& Gorgas (1995) report $\mathrm{Mg}_{2}(\mathrm{mag}) \simeq 0.066 \mathrm{Mgb}(\mathrm{EW})$. The comparison with FFI95 data is shown in Fig. 6. The measurements for the central galaxy in A 779 exhibit an excellent agreement. However, this is not the case for the central galaxies of A 496, A 2199 and A 2634.

The discrepancies in A 496 and A 2199 are readily understood in terms of contamination by the emission line [N $\mathrm{N}] \lambda 5199$. Unfortunately, this line is centred in the red continuum bandpass of the $\mathrm{Mgb}$ index, and its presence translates into an overestimation of the index value due to an enhancement of the measured continuum level. The effects of this emission have been studied in detail by Goudfrooij \& Emsellem (1996). Since in Fig. 6 we have derived $\mathrm{Mg}_{2}$ from the $\mathrm{Mgb}$ indices of FFI95, these $\mathrm{Mg}_{2}$ values are also overestimated. Although we cannot correct the FFI95 indices for the effect of the emission line, we can reproduce their measurements by measuring $\mathrm{Mgb}$ in our spectra without removing the contamination of [NI] $\lambda 5199$, and then transforming these $\mathrm{Mgb}$ values into $\mathrm{Mg} 2$ indices. The effect of such a procedure is shown in Figs 6(b) and (c) as dotted arrows. It is clear from the figures that in this case the agreement between FFI95 data and our gradients is fairly good for A 2199 and A 496. In fact our indices lie between those determined by FFI95 along the major and minor axes.
It is important to note that the effect of [N $\mathrm{N}] \lambda 5199$ in $\mathrm{Mg}_{2}$ is much smaller than in Mgb. Goudfrooij \& Emsellem (1996) showed that a strong EW of [ $\left.\mathrm{N}_{\mathrm{I}}\right] 5199 \sim 1.8 \AA$ translates into $\Delta \mathrm{Mgb} \sim 2$ but only $\Delta \mathrm{Mg}_{2} \sim 0.03$ (transforming this $\Delta \mathrm{Mgb}$ into $\Delta \mathrm{Mg}_{2}$ using the linear relation described above gives $\Delta \mathrm{Mg}_{2} \sim 0.13$, which is four times larger).

Nevertheless, the $\mathrm{Mg}_{2}$ gradient for the central galaxy of A 2634 does not show the same agreement found in the previous objects (note that, in this case, $\mathrm{Mg}_{2}$ is not affected by the emission lines). Our measurements are clearly below the FFI95 data. Additional central $\mathrm{Mg}_{2}$ measurements for A 779, A 2199 and A 2634 are also available from Trager (1997) - plotted as a short horizontal full line - and Lucey et al. (1997) - short horizontal dashed line. These central indices agree with our central values for A 779 and A 2199, confirming the previous comparison with the FFI95 data. In addition, the central $\mathrm{Mg}_{2}$ values of A 2634 from Trager (1997) and Lucey et al. (1997) lie in the region between the FFI95 data and ours. This discrepancy, which affects mainly the absolute values of the $\mathrm{Mg}_{2}$ index, but not the shape of the radial gradient, is not understood, so caution is needed when interpreting the A 2634 data.

\section{DISCUSSION}

\subsection{Central spectral indices}

Different authors have reported correlations of central blue colours and spectral features with mass deposition rates: JFN87 found a correlation between $\mathrm{D}_{4000}$ and the mass flow rate $\left(\dot{M}_{\mathrm{V}}\right)$ within their spectrograph slit; MO89, MO92 and McNamara (1997) shown that central $U-B$ colour excesses in CCGs immersed in clusters with high mass deposition rates also correlate with $\dot{M}$; in CGA95 we confirmed the correlation with $\mathrm{D}_{4000}$ and presented a correlation with $\mathrm{Mg}_{2}$.

In Fig. 7 we have plotted all the central measurements listed in Table 3, as a function of the X-ray derived mass deposition rate. In Figs 7(a) and (b) we have used the total $\dot{M}$ values, whereas in Figs 7(c) and (d) we computed the central mass deposition rates in the 


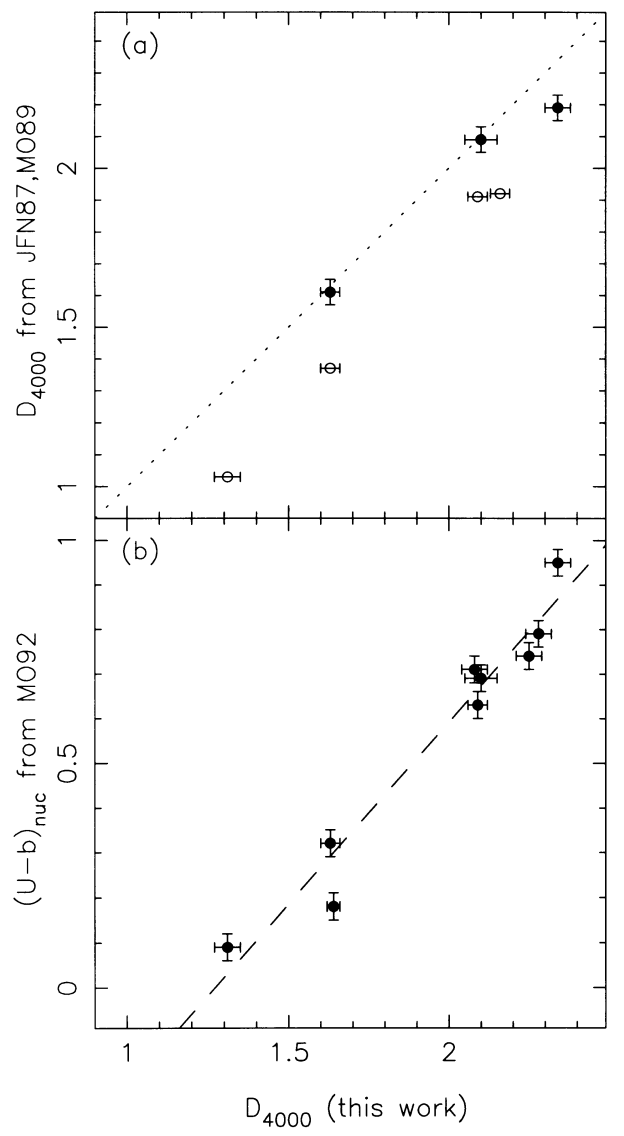

Figure 5. Panel (a): comparison of central $D_{4000}$ measurements from JFN87 - open circles - and MO89 - filled circles - with our central $D_{4000}$ indices for four and three galaxies in common, respectively. Panel (b): correlation between the nuclear $(U-b)$ colours from MO92 with the central $\mathrm{D}_{4000}$ values.

same fixed metric aperture size employed in the measurement of the central indices, assuming $\dot{M} \propto r$. We have also included in Figs 7(a) and (c) central $\mathrm{D}_{4000}$ estimates of eight additional galaxies obtained from the nuclear $(U-b)$ colours of MO92. For this purpose we have used the linear fit derived in Section 4.3. These new indices are listed in Table 5. Filled and open symbols in Fig. 7 correspond to CCGs with and without central emission lines, respectively.

Although, as mentioned above, central $\mathrm{D}_{4000}$ and $\mathrm{Mg}_{2}$ indices are well correlated with mass deposition rate in the sense that both indices decrease when the mass flow increases, it is interesting to note that some objects lie off the main relation. In particular, the cooling flow galaxies without emission lines, A 644, A 2029 and A 2142, are found above the relation, their central indices being fully consistent with those of central galaxies in clusters without cooling flows. Simultaneously, there are also two cooling flow galaxies with emission lines, Hydra A and N 1275, which also seem to depart from the relation with $\dot{M}$.

To perform a simultaneous study of the variations in $\mathrm{D}_{4000}$ and $\mathrm{Mg}_{2}$, we present an index-index diagram in Fig. 8. In Fig. 8(a) we plot the central indices with their errors for the whole sample. In the following panels of this figure we have suppressed the galaxy names and error bars for clarity. Since the CCGs exhibit a clear sequence in this index-index plane, in Fig. 8(b) we compare this sequence with that displayed by early-type galaxies. The small dots correspond to a sample of 135 elliptical and S0 galaxies observed by Kimble,
Davidsen and Sandage (1989; note that we have transformed their $\mathrm{Mgb}$ data into $\mathrm{Mg}_{2}$ using the same relation employed in Section 4.3). As already shown in CGA95, CCGs in cooling flow clusters populate the $\mathrm{D}_{4000}-\mathrm{Mg}_{2}$ plane following a different sequence to that displayed by elliptical galaxies. The enlargement of the galaxy sample allows us to gain new insights into this study. In particular, it is clear from the figure that the CCGs in clusters without cooling flows (open circles) lie in the same region as the giant ellipticals (high $\mathrm{Mg}_{2}$ and $\mathrm{D}_{4000}$ values). The central galaxies in clusters with cooling flows which do not exhibit emission lines in their central parts (open stars, with symbol size proportional to mass deposition rate) are also compatible with the location of the noncooling flow galaxies, even for high $\dot{M}$, like A 2142 and A 2029. On the other hand, cooling flow galaxies with central emission lines (filled circles, with symbol size proportional to $\dot{M}$ ) depart from this location, the largest accretors being the galaxies which show the largest deviations. Using the same symbols, we plot in Fig. 8(c) the $\mathrm{D}_{4000}$ obtained from $(U-b)_{\text {nuc }}$ colours of MO92 (as explained above). It is interesting to note that some cooling flow galaxies with only moderately high $\dot{M}$ (Hydra A and N 1275) display quite low $\mathrm{D}_{4000}$ values. Thus, it is clear that cooling flow galaxies with emission lines do not represent a simple one-parameter sequence in mass deposition rate.

We investigate now the behaviour of the spectral indices as a function of age and metallicity by studying the evolution of single bursts of star formation. We have used the galaxy isochrone synthesis spectral evolution library, GISSEL96, from Bruzual \& Charlot (in preparation). In Fig. 8(d) we plot the evolution of three single bursts of star formation (full lines) with different metallicities ([Fe/H] $=-0.64,-0.33$ and 0.09 ). The model sequences are labelled with small circles which indicate the age of the burst in Gyr. $\mathrm{Mg}_{2}$ values in the models are computed using the empirical fitting functions derived by Gorgas et al. (1993) and Worthey et al. (1994). Unfortunately, this type of fitting function is not available for the $\mathrm{D}_{4000}$, and a non-negligible systematic offset between the predictions of the models and the data could be present. For simplicity, we represent the sequence of normal ellipticals and S0 of Kimble et al. (1989) by a dashed parallelogram. Taking into account the models, we have drawn two vectors to indicate changes of age and metallicity in this $\mathrm{D}_{4000}-\mathrm{Mg}_{2}$ plane and, interestingly, both parameters are not completely degenerate in this diagram. It suggests that normal ellipticals represent a metallicity sequence, whereas the cooling flow objects describe a locus roughly parallel to the age evolution. This strongly suggests that the observed spectral properties of the cooling flow galaxies can be understood as a consequence of star formation.

Following the procedure described in Gorgas et al. (1990) and in CGA95, we have modelled the expected changes in the central $\mathrm{D}_{4000}$ and $\mathrm{Mg}_{2}$ indices when a normal $\mathrm{CCG}$, initially without cooling flow, undergoes star formation as a result of the cooling flow (Fig. 8e). All the models describe the effect of such star formation superimposed on a galaxy with $\mathrm{D}_{4000}$ and $\mathrm{Mg}_{2}$ indices equal to the average values obtained with our subsample of five CCGs in clusters without cooling flows (i.e. typical of old stellar populations). Dotted lines represent the effect of continuous star formation during the last $0.1,1.0$ and $10.0 \mathrm{Gyr}$ (from bottom to top). Full lines correspond to single bursts that occurred $0.01,0.1$ and 1.0 Gyr ago (also from bottom to top). In all these models a Scalo (1986) IMF and solar metallicity were adopted. Finally, the dashed line indicates the effect of a power-law spectrum $\left(F_{\lambda} \propto \lambda^{\alpha-2}\right.$, with $\alpha=0.5$ ). No intrinsic reddening has been taken into account in the models. Small dots in the model lines indicate the fixed fraction $f_{V}$ 

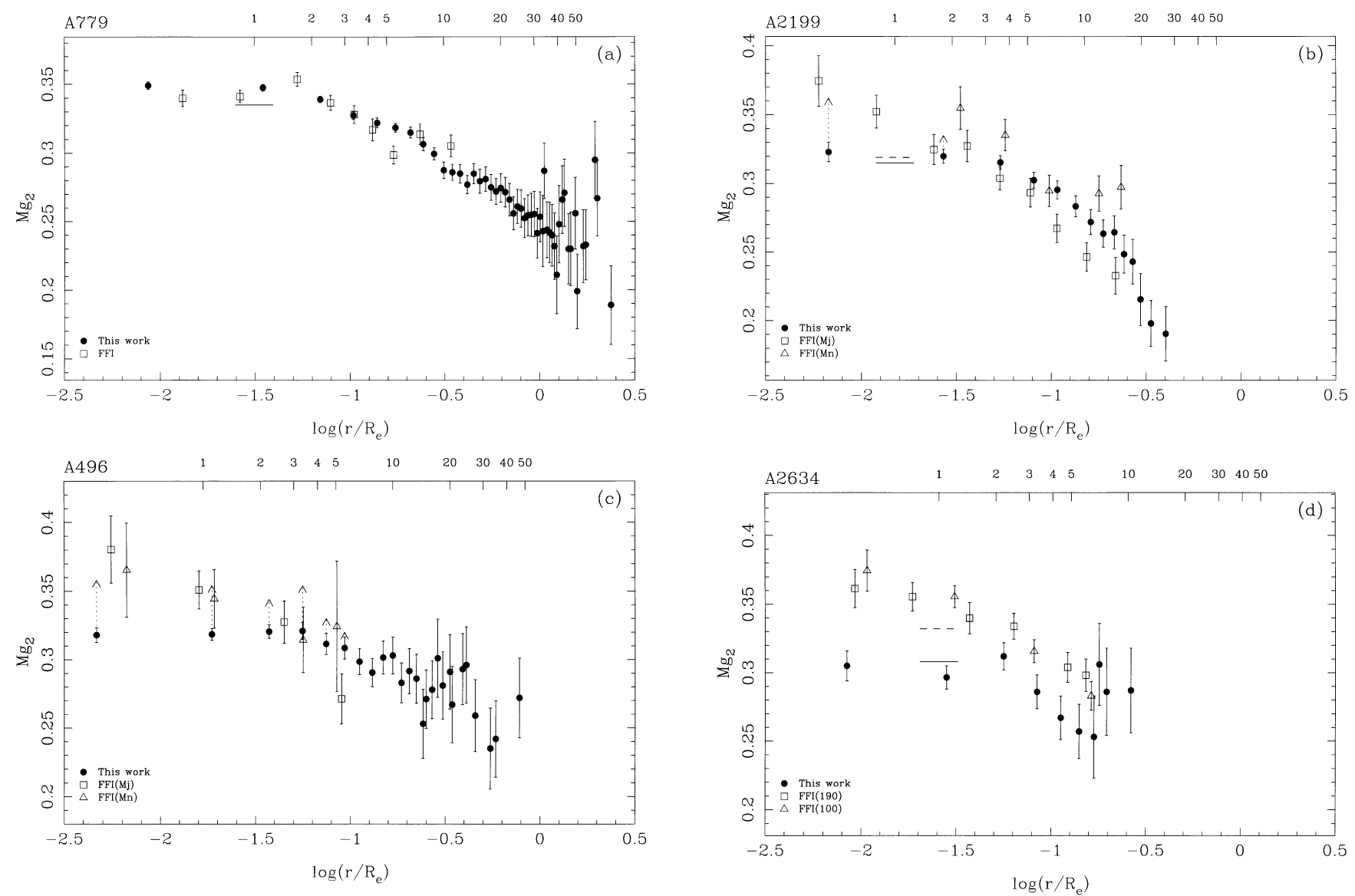

Figure 6. Comparison of $\mathrm{Mg}_{2}$ gradients for four galaxies in common with FFI95. Open symbols (squares: major axis, triangle: minor axis) are from FFI95, whereas filled circles correspond to measurements from CGA95 and this work (major axis, averaged at both sides of the galaxies). The minor axis data from FFI95 are rescaled to reproduce the same spatial scale as the major axis data. The upper $x$ scale in the plots indicates the spatial scale in arcsecs. The atomic Mgb measurements of FFI95 were transformed into molecular $\mathrm{Mg}_{2}$ values and then converted to the Lick system by applying an offset of $0.014 \mathrm{mag}$. This offset has been derived from a comparison of the FFI95 data with a compilation of gradients by González \& Gorgas (1997). Our $\mathrm{Mg}_{2}$ indices were also transformed to the Lick system using the systematic offsets determined through the observation of template stars from the Lick library (see text). The short horizontal full lines at $r \sim 1$ arcsec in panels (a), (b) and (d) correspond to the central $\mathrm{Mg}_{2}$ measurement given by Trager (1997). Similarly, the short horizontal dashed lines in panels (b) and (d) are the central $\mathrm{Mg}_{2}$ values measured by Lucey et al. (1997). The dotted arrows in the inner regions of the central galaxies of A 2199 and A 496 indicate the location of our $\mathrm{Mg}_{2}$ indices if we had measured $\mathrm{Mgb}$ in our spectra without removing the contamination by the emission line [ $\left.\mathrm{N} \mathrm{I}\right] \lambda 5199$, and then transformed these Mgb values into $\mathrm{Mg}_{2}$ (in the same way as we did with the FFI95 data). The effect of the emission lines in A 2634 is completely negligible.

(given by the numbers) of the $V$ light that comes from the accretion population (i.e. the newly formed stars). In the case of continuous star formation, this fraction $f_{V}$ can be related to the SFR using the expression

$S F R=\frac{f_{V} L_{V}(M / L)_{\mathrm{AP}}}{t}$,

where $L_{V}$ is the total $V$ luminosity of the galaxy, $(M / L)_{\mathrm{AP}}$ is the stellar mass-to-light ratio of the accretion population, given by the models, and $t$ is the time over which the star formation has taken place. Similarly, the parametrization for the models involving a single burst of star formation is given by

$M_{\mathrm{AP}}=f_{V} L_{V}(M / L)_{\mathrm{AP}}$,

where $M_{\mathrm{AP}}$ is the total mass in stars formed in the accretion population.

Fig. 8(e) reveals that the sequence of cooling flow galaxies does not seem to be very well reproduced by continuous star formation during the last 1-10 Gyr. Either a young (<1.0 Gyr) continuous star formation, or simply a recent burst of star formation $\sim 0.1 \mathrm{Gyr}$ ago, can match the observed central indices. In the lower right corner of Fig. 8(e) we have also plotted the effect of intrinsic reddening in the measurements. As an example, we represent the corrections for an hypothetical object with $\mathrm{D}_{4000}=1.7$ and $\mathrm{Mg}_{2}=0.300$ as a function of $E(B-V)$ (the colour excess ranges from 0.0 to 1.0 , with ticks spaced 0.1 units). As it could be expected, the effect on $\mathrm{Mg}_{2}$ is almost negligible, whereas the contrary is true for the $\mathrm{D}_{4000}$. Although Hansen et al. (1995) have reported $E(B-V) \leq 0.1$ for Hydra A, other authors have found non-negligible reddening values $[E(B-V) \sim 0.1-0.6]$ for several cooling flow galaxies (e.g. Hu 1992; Allen et al. 1995; Allen 1995; Donahue \& Voit 1993; Voit \& Donahue 1997). If we allow for the existence of internal reddening, the case for recent bursts instead of continuous star formation is even stronger. Nevertheless, it is important to keep in mind that the effect of reddening in the stellar continuum is usually lower than in the emission lines, e.g. Calzetti (1997) reports $E(B-V)_{\text {stellar }}=0.44 E(B-V)_{\text {gas }}$ in typical starforming regions.

However, we need to be cautious since, apart from intrinsic reddening, there are additional sources of uncertainty present in the models. First, there is some uncertainty in the starting point of the 


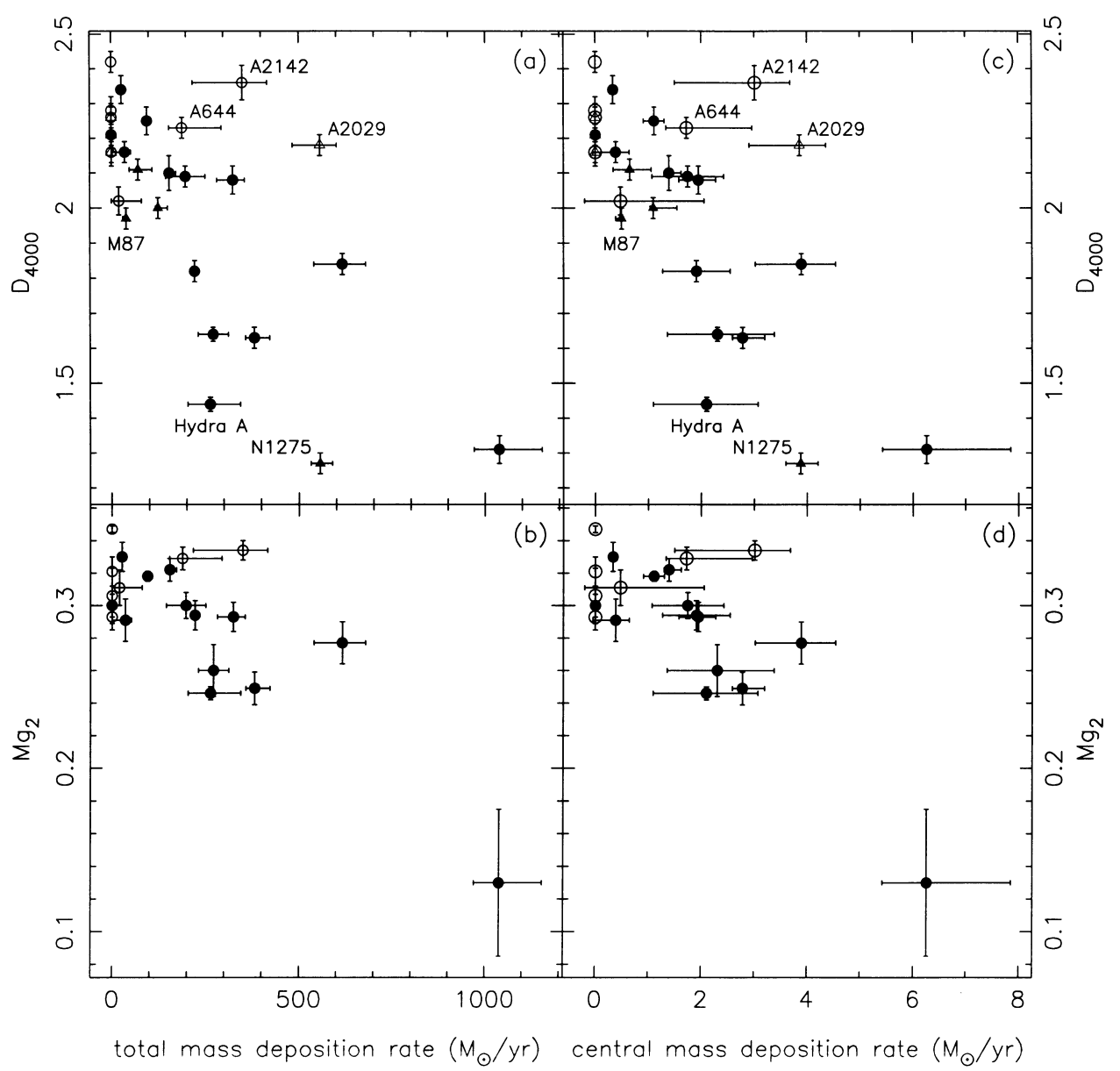

Figure 7. Central $\mathrm{D}_{4000}$ and $\mathrm{Mg}_{2}$ measurements versus X-ray-derived mass deposition rates (the sources for $\dot{M}$ are given in Table 3 ). The central indices were obtained by employing a fixed metric aperture size of 4 arcsec projected at the distance of the Coma cluster. Central mass deposition rates have been computed from the total mass deposition rates assuming $\dot{M} \propto r$. Error bars in the mass deposition rate indicate the 10 and 90 percentile estimates. Filled and open symbols correspond to CCGs with and without emission lines in the central regions, respectively. Circles are galaxies from CGA95 and this paper, whereas triangles indicate $\mathrm{D}_{4000}$ values derived from nuclear $(U-b)$ colours published by MO92, as explained in the text.

Table 5. Central $\mathrm{D}_{4000}$ indices derived from the nuclear $(U-b)$ colours published by MO92, using the linear relation fitted in Fig. 5(b). Errors $\left(\Delta \mathrm{D}_{4000}\right)$ have been derived from the $(U-b)$ errors reported by MO92. Sources for mass deposition rate and cooling radius are those quoted in Table 3.

\begin{tabular}{|c|c|c|c|c|c|c|c|}
\hline Cluster & Galaxy & $D_{4000}$ & $\Delta \mathrm{D}_{4000}$ & $\begin{array}{c}\dot{M} \\
\left(\mathrm{M}_{\odot} \mathrm{yr}^{-1}\right)\end{array}$ & $r_{\text {cool }}$ & $\begin{array}{l}\text { central } \dot{M} \\
\left(\mathrm{M}_{\odot} \mathrm{yr}^{-1}\right)\end{array}$ & $\begin{array}{c}\text { source of } \\
\dot{M}, r_{\text {cool }}\end{array}$ \\
\hline A 119 & $\ldots$ & 2.16 & 0.03 & $0_{-0}^{+2}$ & $0_{-0}^{+62}$ & $0.00{ }_{-0.00}^{+0.00}$ & 1 \\
\hline A 426 & N 1275 & 1.27 & 0.03 & $556_{-24}^{+33}$ & $185_{-11}^{+11}$ & $3.88_{-0.17}^{+0.23}$ & 1 \\
\hline MKW 1 & N 3090 & 2.26 & 0.03 & 0 & $\ldots$ & $\ldots$ & 3 \\
\hline Virgo & M 87 & 1.97 & 0.03 & $39{ }_{-9}^{+2}$ & $102_{-4}^{+6}$ & $0.49{ }_{-0.11}^{+0.03}$ & 1 \\
\hline MKW 5 & N 5400 & 2.21 & 0.03 & 0 & $\ldots$ & $\ldots$ & 3 \\
\hline A 1991 & N 5778 & 2.11 & 0.03 & $71_{-23}^{+38}$ & $142_{-48}^{+48}$ & $0.65_{-0.21}^{+0.34}$ & 5 \\
\hline А 2029 & IC 1101 & 2.18 & 0.03 & $556_{-73}^{+44}$ & $186_{-39}^{+19}$ & $3.86_{-0.51}^{+0.31}$ & 1 \\
\hline A 2052 & $\ldots$ & 2.00 & 0.03 & $125_{-6}^{+26}$ & $147_{-3}^{+53}$ & $1.09_{-0.05}^{+0.22}$ & 1 \\
\hline
\end{tabular}

model sequences, since the dispersion in the central indices of CCGs without cooling flows is probably real. Secondly, we have already mentioned that the modelling of $\mathrm{D}_{4000}$ is somewhat uncertain given the lack of empirical calibrations, thus some systematic effects could be present. And finally, the models assume solar metallicity and solar element ratios: if there is an enhancement of
$[\mathrm{Mg} / \mathrm{Fe}]$ in the cooling flow material, the predicted $\mathrm{Mg}_{2}$ indices could be underestimated. For these reasons, it seems premature to rule out competely, from the models shown in Fig. 8(e) alone, continuous star formation (lasting some Gyr) as the source of the blue light.

Considering the relatively short-wavelength baseline between 


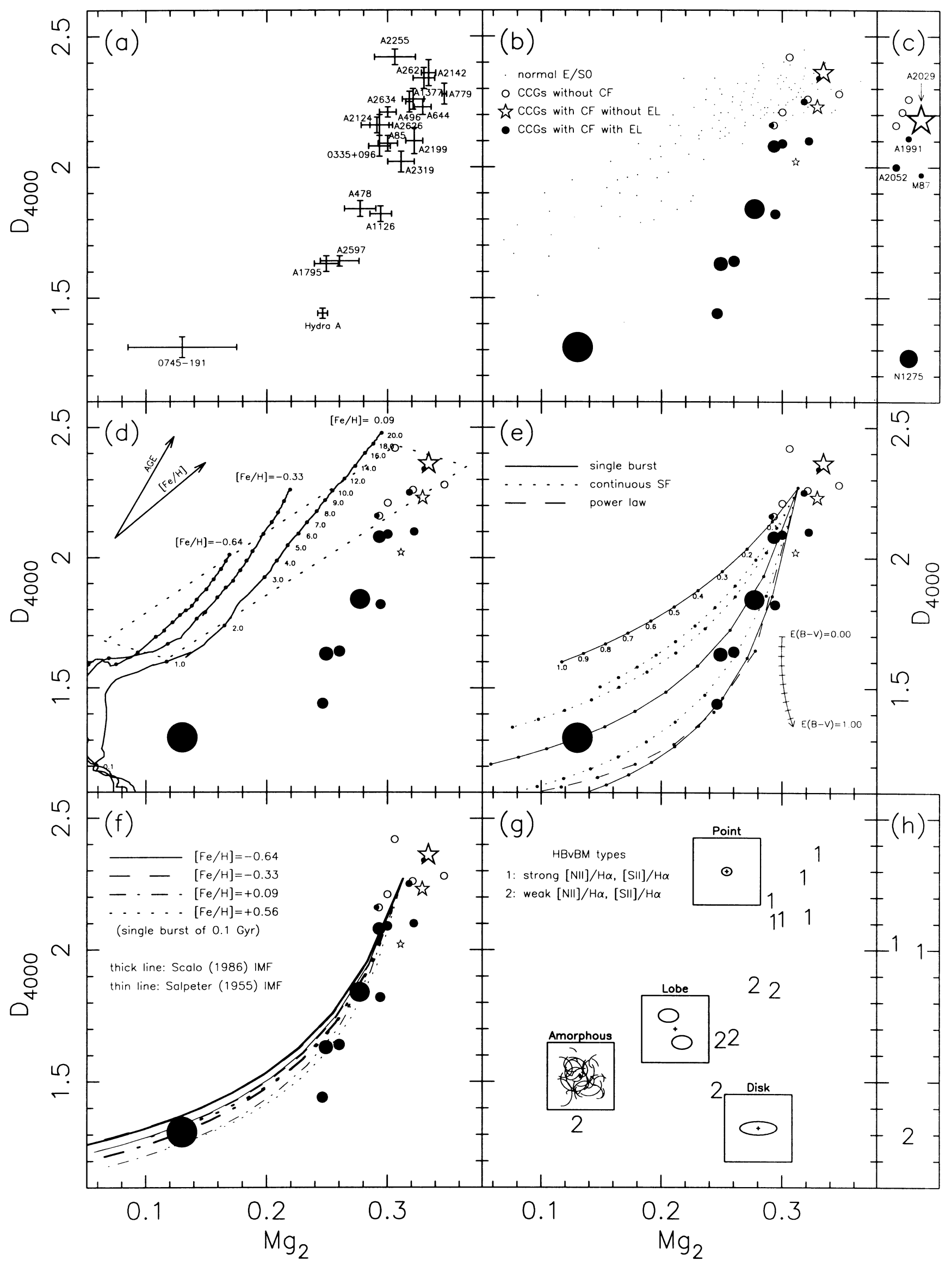

Figure 8. Central $\mathrm{D}_{4000}$ versus $\mathrm{Mg}_{2}$ measurements. See explanation in Section 5.1. 


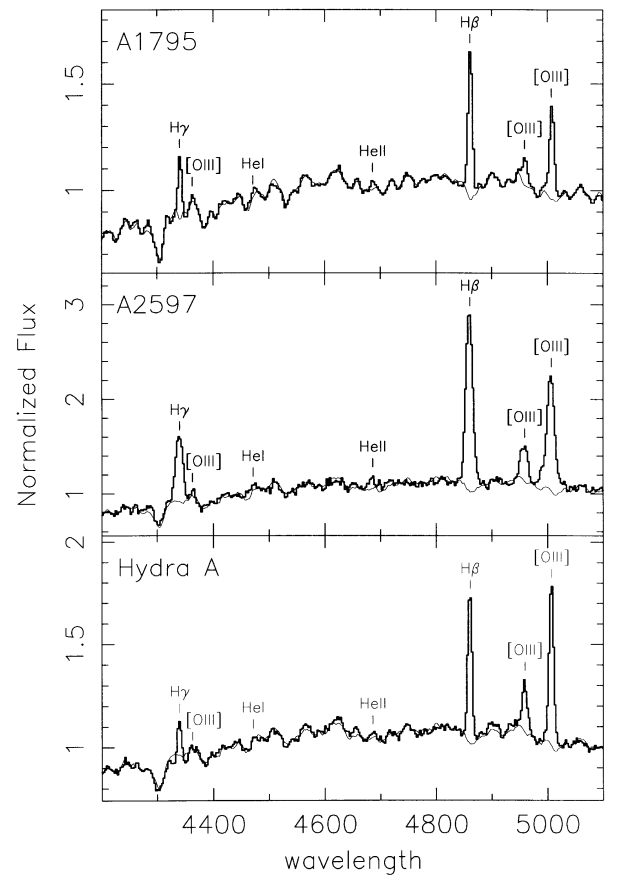

Figure 9. Central spectrum of three CCGs of our sample with emission lines (A 1795, A 2597 and Hydra A, from top to bottom, thick line), compared with scaled template spectra (+low-order polynomial) obtained from the central regions of CCGs without emission lines (A 2124, A 644 and A 644, from top to bottom, thin line). A marginal detection of He II $\lambda 4686$ is present in A 1795 and in A 2597, whereas this is uncertain in the case of Hydra A.

the $\mathrm{D}_{4000}$ and $\mathrm{Mg}_{2}$ indices, the contribution of a power-law spectrum could also reproduce the observed spectral changes. This result is not surprising since, in a short wavelength interval, the spectrum of an early-type star can be approximated quite well by a power law (see also Crawford \& Fabian 1993). However, as we have already discussed in the introduction, the absence of polarization in the blue light of A 1795 (McNamara et al. 1996b) does not give strong support to this alternative. In addition, some central galaxies show clear signatures of strong Balmer absorption lines (e.g. N 1275, Crawford \& Fabian 1993; Hydra A, Hansen et al. 1995; Melnick et al. 1997). Allen (1995) found that the strong UV/ blue continua in the spectra of a small sample of CCGs were better described by young stars than by power-law emission models. This author also reported positive detection of Wolf-Rayet features in two CCGs (those in A 1068 and A 1835), which favour the idea of the formation of massive stars. In this sense, Voit \& Donahue (1997) have measured a weak He II $\lambda 4686$ emission in A 2597 . We also find evidences of such helium feature in the central spectrum of the dominant galaxies in A 1795 and A 2597 (see Fig. 9)

The role of metallicity and the choice of IMF on the model predictions plotted in Fig. 8(e) is explored in Fig. 8(f). We show the GISSEL96 (Bruzual \& Charlot, in preparation) predictions for different metallicities (as explained in the panel legend), and two IMFs, namely Salpeter (1955) - thin lines - and Scalo (1986) thick lines, for the single burst model with an age of $0.1 \mathrm{Gyr}$. The predictions of the models do not depend strongly on the choice of either $[\mathrm{Fe} / \mathrm{H}]$ or the IMF.

In order to obtain further constraints on the range of acceptable models, we need to explore a larger wavelength range. We have done so using UV and near-IR data from the literature (see Fig. 10 and description in the figure caption). Although continuous star formation lasting $10 \mathrm{Gyr}$ could explain the observed optical and near-IR observations, the UV fluxes are underpredicted. Recent bursts with ages $\sim 0.1-0.2$ Gyr are simultaneously compatible with both the UV and IR fluxes, and with the measured central linestrength indices. Note, however, that at UV wavelengths the effects of dust extinction can be quite dramatic even for moderate colour excesses: using the interstellar reddening curve of Savage \& Mathis (1979), and assuming $E(B-V)=0.2$ and $E(B-V)=0.4$, we estimate that the measured UV fluxes need to be corrected upwards by factors of $\sim 5-6$ and $\sim 20-30$, respectively. In these cases, is quite obvious that the UV continuum would constrain the upper limit of the burst age to be $<0.1 \mathrm{Gyr}$. This result agrees with the behaviour of $\mathrm{D}_{4000}$ and $\mathrm{Mg}_{2}$ with $E(B-V)$ in Fig. 8(e), indicating that a recent burst would be strongly favoured against constant star formation.

Another piece of evidence which can help to understand the behaviour of the spectral indices of the CCGs comes from the emission-line properties. Using line-ratio diagrams $\mathrm{HBvBM}$ classified the emission nebulae at the centres of central dominant galaxies into two types. In particular, Type I corresponds to objects with relatively high $\left[\mathrm{N}_{\mathrm{II}}\right] \lambda 6584 / \mathrm{H} \alpha(\simeq 2.0)$ and $\left[\mathrm{S}_{\mathrm{II}}\right] \lambda 6717 / \mathrm{H} \alpha$ $(\simeq 0.7)$, whereas Type II objects exhibit lower line ratios ([N $\mathrm{II}] \lambda 6584 / \mathrm{H} \alpha \simeq 0.9$ and $\left[\mathrm{S}_{\mathrm{II}}\right] \lambda 6717 / \mathrm{H} \alpha \simeq 0.4$ ). In panels $8(\mathrm{~g})$ and (h) we have plotted the available HBvBM types for the galaxies in our sample and in MO92 respectively. There is a clear dichotomy in the way Type I (plotted with ' 1 ' symbols) and Type II (plotted with ' 2 ' symbols) galaxies populate the central cluster galaxy sequence. This result is not surprising since Type I objects are associated with clusters with low X-ray and $\mathrm{H} \alpha$ luminosities, small optical nebulae and low mass deposition rates, whereas the contrary is true for Type II objects (HBvBM; Baum 1992; Donahue \& Voit 1997).

Another clue comes from the morphology of the blue excess light. Recently, McNamara (1997) has established a morphological classification based on the study of the surface brightness distributions and colour structure in cooling flow CCGs. This classification consists of four morphological types ordered by increasing geometrical complexity:

(1) point source (e.g. A 2199, A 2052) with unresolved blue nuclei and nebular line emission, low nebular luminosity, with modest blue anomalies;

(2) disc (e.g. Hydra A), unusual in cooling flow galaxies and characterized by a disc of gas and young stars rotating around the nucleus;

(3) lobe (e.g. A 1795, A 2597) with bright, blue lobes of optical continuum separated several kiloparsecs from the nucleus; and

(4) irregular-amorphous (e.g. N 1275 may be in transition between types 3 and 4 ).

In Fig. 8(g) we have also plotted the four morphological types in the neighbourhood of the galaxies which display such characteristics. Taking into account the $\mathrm{D}_{4000}$ exhibited by $\mathrm{N} 1275$, we have tentatively located Type 4 near PKS0745 - 191. Very interestingly, McNamara (1997) has speculated about the possibility that the structural types actually reflect an evolutionary sequence. We will discuss this point in more detail in Section 6.

\subsection{Line-strength gradients}

Although line-strength gradients in $\mathrm{Mg}_{2}$ (e.g. Faber 1977; Gorgas et al. 1990; González 1993; Davies et al. 1993; Carollo et al. 1993; FFI95; González \& Gorgas 1995) and in $\mathrm{D}_{4000}$ (e.g. Munn 1992; 


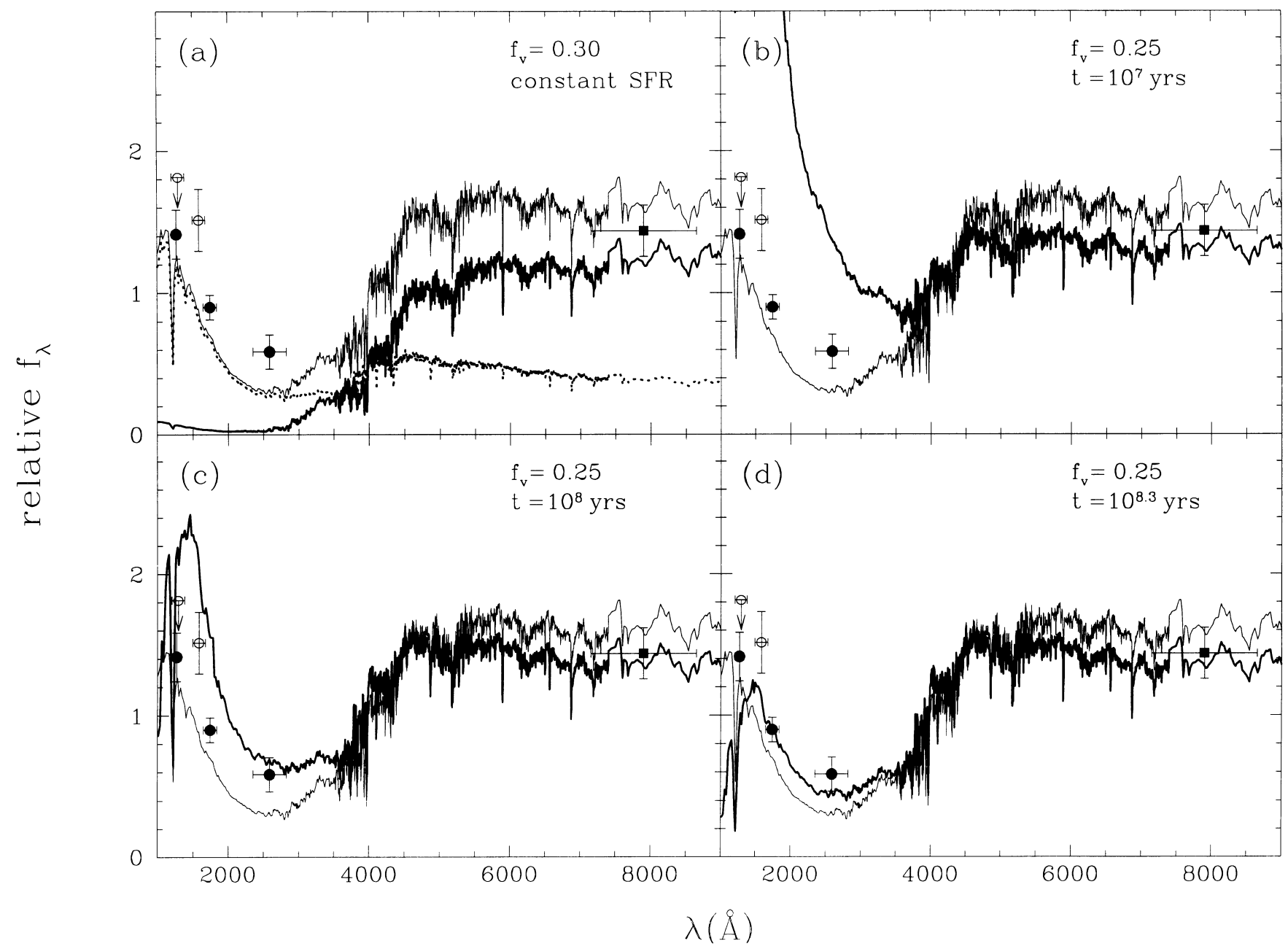

Figure 10. Modelling of different star formation episodes superimposed on the spectrum of an old stellar population. We have employed the GISSEL96 predictions (Bruzual \& Charlot 1997), with solar metallicity and Scalo (1986) IMF. Circles are relative UV fluxes from Crawford \& Fabian (1993) (filled circles: A 1795, open circles: A 2597), whereas the filled square is an IR flux estimation from the averaged nuclear $(U-I)$ colours of A 1795 and A 2597 reported by MO92. Panel (a) shows the spectral energy distribution (SED) corresponding to an old (15 Gyr) stellar population (thick line), continuous star formation during the last $10 \mathrm{Gyr}$ (dotted line), and the final product of both stellar components (thin line) assuming that the fraction of $V$ light that comes from the continuous star formation is $f_{V}=0.3$. The prediction of this continuous star formation (thin line) is compared, in the following panels, with the final SEDs obtained by superimposing a single burst of star formation on the spectrum of the old stellar population. In particular, we have represented the resulting spectrum (thick line $=15$ Gyr stellar population + single burst, $f_{V}=0.25$ ) when $0.01,0.1$ and $0.2 \mathrm{Gyr}$ have elapsed after the time the burst took place [panels (b), (c) and (d) respectively]. See discussion in the text.

Davidge \& Grinder 1995) are usually found to be linear with $\log (r)$ in elliptical galaxies, in CGA95 we showed that some cooling flow galaxies exhibit a clear slope change at intermediate radii. With the inclusion of the new data presented in this paper (see Figs 3 and 4), we find that this change of slope is clearly related to the presence of emission lines in the central regions of CCGs.

We measured the radius at which the change of slope takes place using error-weighted least-squares fits to two straight lines forced to join at a variable radius. The method gives the break radius which minimizes the residual variance of the fits. We estimated the uncertainty in the break radius using Monte Carlo simulations that take into account the measurement errors in the indices (typically $\simeq 1000$ simulations per gradient). For the emission-line galaxies with reasonably good data beyond the emission region, we have compared in Fig. 11 the break radius with the observed emission-line radius (as defined in Section 4.2). The figure reveals that the break radii are almost coincident with the emission-line radii, with the former being slightly larger.
It is quite evident from Figs 3 and 4 (see also the fitted gradients tabulated in Table 4), that cooling flow galaxies with emission lines exhibit flat and even positive gradients in the region where the emission is detected (e.g. A 478, A 1795, A 2597, Hydra A, A 85, A 2199). In fact, these inner gradients (measured in the emissionline region) seem to be correlated with the mass deposition rate, i.e. more positive with increasing $\dot{M}$ (see Figs $12 \mathrm{a}$ and b). In the outer parts of these galaxies, where emission is not observed, the derived mean $\mathrm{D}_{4000}$ gradient is consistent with that observed in normal elliptical galaxies (see Fig. 12c). However, the mean $\mathrm{Mg}_{2}$ gradient in the outer regions of cooling flow galaxies with emission lines seems to be marginally steeper than the mean gradient for normal ellipticals (Fig. 12d), although the quality of the $\mathrm{Mg}_{2}$ data at large galactocentric distances is not good enough to reach a firm conclusion. A couple of galaxies (A 1126 and A 262) seem to have slightly steeper gradients in the inner parts than in the outer regions, although this result is not very significant due to the poor $\mathrm{S} / \mathrm{N}$ in the outer spectra. In addition, mean gradients in central dominant 


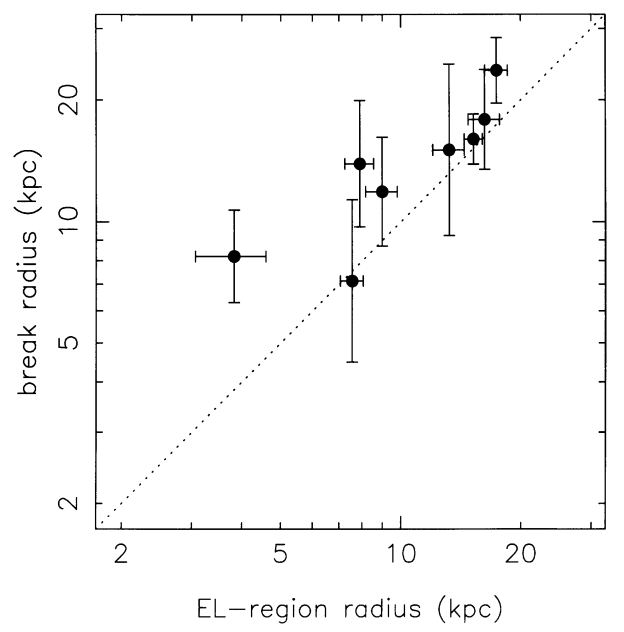

Figure 11. Comparison of the break radius obtained through the simultaneous fit of two straight lines forced to join at a common radius, with the radius of the emission-line region. Break radii and their error bars correspond to the mean and standard deviation derived in Monte Carlo simu lations. When available, estimates of the break radius from $\mathrm{D}_{4000}$ and $\mathrm{Mg}_{2}$ line-strength gradients have been averaged. Emission-line radii were determined in the spectroscopic images as the radii where the emission lines are no longer detectable (see Section 4.2). galaxies with cooling flows but without emission lines (e.g. A 644, A 2142) are similar to those in galaxies without cooling flows and in elliptical galaxies.

If we assume that the observed slope changes in the line-strength gradients are due to radial index variations induced by star formation in the emission-line region, we can use that information, coupled with the star formation models, to estimate the amount and spatial profile of the accretion population. We assume that the gradients determined in the outer regions represent the underlying gradient, i.e. that of the galaxy before the cooling-flow-induced star formation takes place. That is justified by the similarity of these outer gradients to those of 'quiescent' galaxies (i.e. both normal giant ellipticals and CCGs without cooling flows). In this analysis we have exclusively concentrated on the $\mathrm{D}_{4000}$ gradients, which are much better determined than the $\mathrm{Mg}_{2}$ ones.

As discussed in Section 5.1, a single burst model with an age $\simeq 0.1 \mathrm{Gyr}$, solar metallicity and Scalo IMF is able to reproduce the observed spectral properties of the cooling flow galaxy sequence. We have employed this particular model to tentatively obtain the spatial profile of the accretion population. First we computed the averaged indices inside the emission-line region (simulating a circular aperture by weighting with light and radius), both from the fitted inner gradient and from the extrapolation of the outer gradient into the emission-line region. Then, we used the star
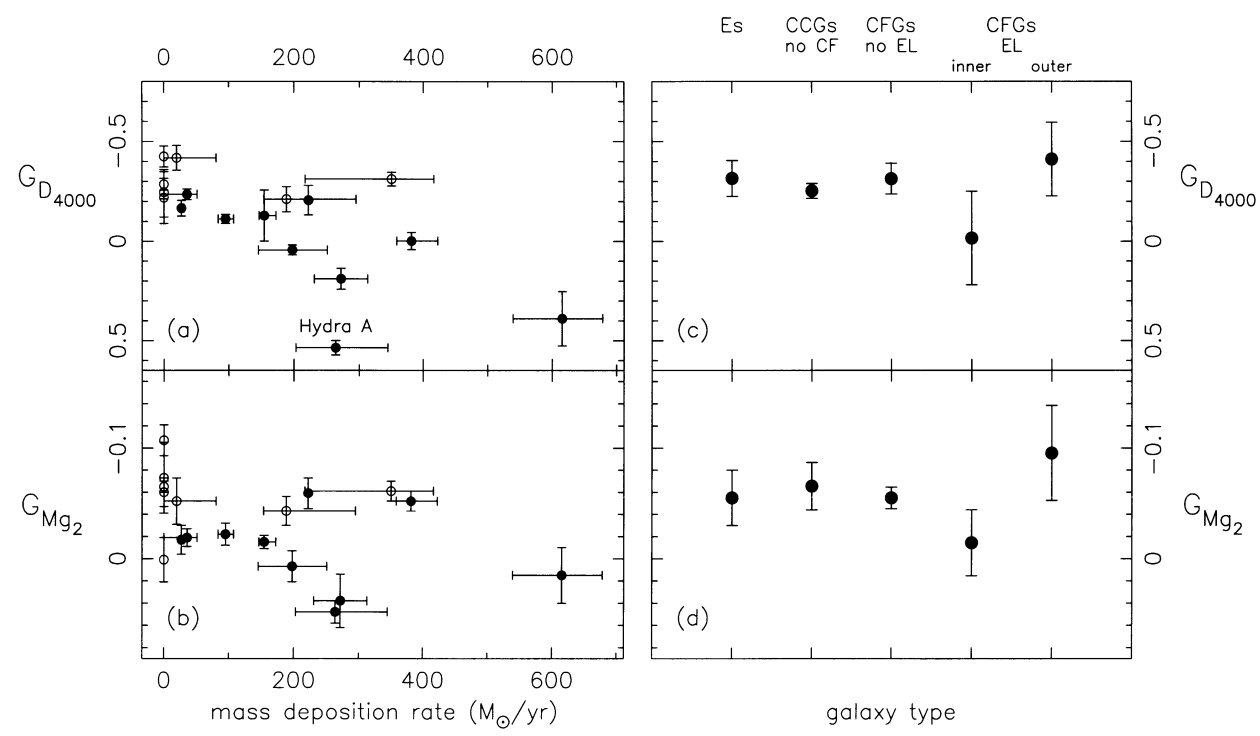

Figure 12. Line-strength gradients in $\mathrm{D}_{4000}$ and $\mathrm{Mg}_{2}$ for the galaxies tabulated in Table 4 as a function of the mass deposition rate - panels (a) and (b). Open symbols are total gradients in galaxies without emission lines. Filled symbols correspond to inner gradients (those measured in the emission-line region). Error bars in mass deposition rate indicate the 10 and 90 percentile estimates. In panels (c) and (d) we compare the mean gradients as a function of the galaxy type: normal ellipticals (Es), central dominant galaxies in clusters without cooling flow (CCGs no CF), and cooling flow galaxies without (CFGs no EL) and with emission lines (CFGs EL). In the latter type we also distinguish between inner and outer gradients, which correspond to the emission-line region and outside this region, respectively. The error bars in the last two panels are the rms scatter around the mean values. The mean $\mathrm{D}_{4000}$ gradient for Es $(-0.315$, rms $=0.090)$ has been computed from the data presented by Munn (1992) and Davidge \& Clark (1994) (see CGA95 for further details). In addition, the Mg $\mathrm{g}_{2}$ gradient for Es $(-0.055, \mathrm{rms}=0.025)$ is the one derived by González \& Gorgas (1997) using published and unpublished data for 109 galaxies with reliable $\mathrm{Mg}_{2}$ profiles.

\footnotetext{
${ }^{2}$ Hoessel, Gunn \& Thuan (1980) reported that the mean magnitude of a sample of brightest cluster galaxies inside a metric radius of $16 / h_{60} \mathrm{kpc}$ is $\left\langle M_{V}\right\rangle=-22.68 \pm 0.03 \mathrm{mag}$. In addition, Hoessel (1980) showed that the internal regions of brightest cluster galaxies are adequately described by a modified Hubble law of the form $I(r)=I_{\mathrm{c}} /\left(1+r^{2} / r_{\mathrm{c}}^{2}\right)$, where $I_{\mathrm{c}}$ is the central intensity, $r_{\mathrm{c}}$ the core radius and $r$ the angular distance from the centre. Assuming this intensity profile, the integrated luminosity inside a radius $r$ is then given by $L(r)=\pi I_{\mathrm{c}} r_{\mathrm{c}}^{2} \ln \left(1+r^{2} / r_{\mathrm{c}}^{2}\right)$. Introducing the value of the Hubble constant adopted in this paper $\left(H=50 \mathrm{~km} \mathrm{~s}^{-1} \mathrm{Mpc}^{-1}\right)$, and the mean core radius derived by Hoessel (1980), $\left\langle r_{\mathrm{c}}\right\rangle=2.23 / h_{60} \mathrm{kpc}$, we obtain $\left\langle M_{V}\right\rangle \sim-22.3$ mag inside a radius $r \sim 11 / h_{50} \mathrm{kpc}$, which is the average radius of the emission-line regions in our sample.
} 


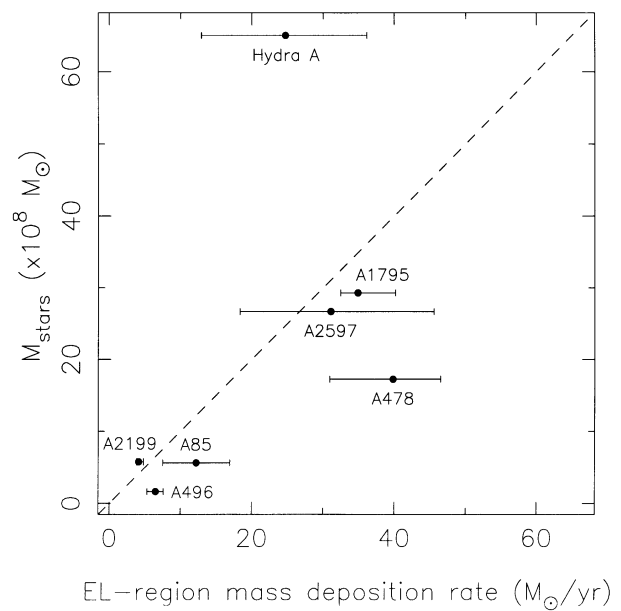

Figure 13. Total mass transformed into stars in the emission-line region of the central galaxies with reliable $\mathrm{D}_{4000}$ gradients in the outer parts. The plotted values have been derived using equation (2), and assuming that a single burst of star formation (after $0.1 \mathrm{Gyr}$ ) has produced the observed $\mathrm{D}_{4000}$ variation corresponding to the difference between the measured inner gradient and the extrapolated outer gradient.

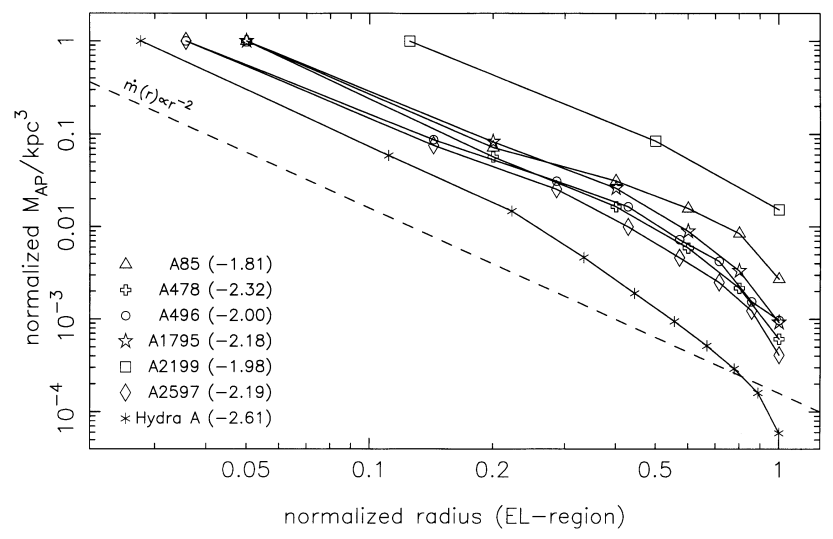

Figure 14. Spatial profile of the mass transformed into stars obtained through the deprojection of the observed radial variations in $\mathrm{D}_{4000}$ (see Appendix A). The dashed line is the expected density of the mass deposition profile is one assumes that $\dot{M}(<r) \propto r$. The numbers in parenthesis are the profile slopes obtained by least-squares linear fits.

formation model to derive the mean $f_{V}$ of the accretion population required to reproduce the observed changes in the indices (i.e. the difference between the measured values and those estimated from the extrapolation of the outer gradient). Assuming that the absolute magnitude corresponding to the old stellar population in the emission-line region is $\left\langle M_{V}\right\rangle \sim-22.3 \mathrm{mag},{ }^{2}$ the mean $f_{V}$ value is then employed to compute the total $V$ luminosity $\left(L_{V}\right)$ of the galaxy plus the burst. Using equation (2), and the mass-to-light ratio of the accretion population given by the model, we obtain the total mass in stars of the accretion population formed in the last burst. Note that, since the excess blue light is completely dominated by the youngest stars, this method is only sensitive to the stars formed in the most recent burst.

In Fig. 13 we have plotted the estimated total amount of mass transformed into stars in the emission-line region versus the mass deposition rate computed in the same area (assuming that $\dot{M} \propto r$ ). There is an increase in the mass transformed into stars with $\dot{M}$, with Hydra A lying somewhat outside this trend. Interestingly, the total mass accreted by the cooling flow in a $\sim 0.1 \mathrm{Gyr}$ period inside the emission-line region is remarkably similar to the mass in new stars (see Section 6).

Assuming spherical geometry, it is possible to apply the same technique to obtain the amount of mass transformed into stars in concentric shells at different radii. Using a deprojection algorithm described in Appendix A, we have derived the spatial profiles for the density of mass transformed into stars (Fig. 14). We also show (dashed line) the expected density profile of the mass deposition assuming $\dot{M}(<r) \propto r$. Interestingly, the derived profiles seem to be quite parallel to the dashed line (the mean slope for all the galaxies excluding Hydra A is -2.08 , rms $=0.18$ ), whereas Hydra A exhibits a statistically significant steeper trend. This result strongly suggests a direct connection between the mass deposition and the star formation profiles. Indeed, Figs 13 and 14 suggest a very close link between the star formation and the cooling flow phenomenon. Such a close connection would be difficult to understand without most of the gas accreted in the emission-line region being transformed into stars.

\section{SUMMARY: TOWARDS A COMPLETE PICT URE?}

Many pieces of the cooling flow puzzle are now available. We summarize some of them here before trying to sort out a scenario able to explain the observational data.

(i) Central $\mathrm{D}_{4000}$ and $\mathrm{Mg}_{2}$ indices in cooling flow galaxies are correlated with mass deposition rate when emission lines are found in the central regions of such galaxies.

(ii) The nuclear indices of the cooling flow galaxies in our sample that do not exhibit emission lines do not follow this correlation with $\dot{M}$. The central line strengths of both CCGs in clusters without cooling flows and cooling flow galaxies without emission lines are consistent with the values observed in giant ellipticals.

(iii) The central $\mathrm{D}_{4000}$ and $\mathrm{Mg}_{2}$ indices of elliptical galaxies define a relatively narrow trend in an index-index plane. Cooling flow galaxies with emission lines exhibit a clear sequence in this diagram which departs from the locus defined by elliptical galaxies. When interpreted using stellar population models, this provides strong evidence for star formation in these galaxies.

(iv) Although line-strength gradients are usually found to be linear with $\log (r)$ in elliptical galaxies, $\mathrm{D}_{4000}$ and $\mathrm{Mg}_{2}$ gradients in cooling flow galaxies with emission lines show a clear slope change in the region where the emission is detected. In fact, these inner gradients seem to be correlated with the mass deposition rate. In the outer parts of these objects, where emission is not observed, the derived mean line-strength gradients are consistent with those in elliptical galaxies.

(v) Mean gradients in cooling flow galaxies without emission lines are similar to those in CCGs without cooling flows and those in giant elliptical galaxies.

(vi) The presence of emission lines must be in some way related to the cooling flow phenomenon since $\mathrm{H} \beta$ (JFN87) and $\mathrm{H} \alpha$ luminosities (HBvBM; Donahue \& Voit 1997) are correlated with mass deposition rate. However, considering the high scatter in line luminosities for a given $\dot{M}$, the relationship does not seem to be a simple one (Baum 1992).

(vii) Whichever mechanism is responsible for the dilution of the measured spectral indices, the correlations of the central indices and gradients with the mass deposition rate in the emission-line region 
add further weight to the idea of a link between the blue excess and the cooling flow. Nevertheless, as Crawford \& Fabian (1993) pointed out, the fact that there are central galaxies in clusters with high $\dot{M}$ but without blue excess suggests that the cooling flow alone cannot be responsible for the blue light. In particular, these authors noted the existence of strong cooling flow galaxies, with (e.g. A 478) and without (e.g. A 2029) emission-line nebulae, which apparently did not exhibit a blue continuum excess. However, our $\mathrm{D}_{4000}$ and $\mathrm{Mg}_{2}$ gradients in the central galaxy of A 478 do exhibit a clear positive behaviour (i.e. blue excess) in the emission-line region. An important question is whether there are any cooling flow galaxies with emission-line nebulae which do not exhibit a blue excess, and vice versa. So far this does not seem to be the case.

(viii) Through a detailed analysis of the emission-line nebula in the central cluster galaxy of A 2597, Voit \& Donahue (1997) conclude that hot stars are the most likely ionizing source of the gas if some mechanical form of heating (flowing from the hot intracluster medium into the cooler nebula) supplements photoelectric heating.

(ix) With the available data, there is a clear dichotomy in the way cooling flow galaxies populate the $\mathrm{D}_{4000}-\mathrm{Mg}_{2}$ plane depending on their emission-line properties as described by the classification scheme of HBvBM. The relative positions of Type I and Type II objects in Fig. 8(g) strongly suggest that recent star formation (and therefore photoionization by hot stars) is the key to understanding the observed dichotomy.

(x) The excess of blue light (i.e. line-strength dilution) in the continuum spectra of CCGs is consistent with recent star formation episodes [e.g. Bertola et al. 1986; JFN87; Shields \& Filippenko 1990; Crawford \& Fabian 1993; Hansen et al. 1995; Allen 1995; Melnick et al. 1997; Figs 8(e) and 10, this work].

(xi) If the observed index variations are due to star formation in the emission-line region, the existence of correlation between the central indices and the line-strength gradients with the mass deposition rates implies that the cool gas must be transformed into stars with a similar efficiency from galaxy to galaxy. In addition, the radial profiles of the star formation density (Fig. 14) are remarkably parallel to the expected density of the mass deposition profile. Both results are difficult to understand unless a large fraction (probably most) of the cooling flow gas accreted into the emission-line region is converted into stars.

(xii) A large fraction (60-70 per cent) of cD galaxies in cooling flows are radio-loud, whereas this probability decreases to 20 per cent for $\mathrm{cD}$ galaxies in non-cooling flow clusters, and 14 per cent for typical elliptical galaxies in clusters (Burns et al. 1997). Since the kinetic energy of the radio plasma can be a significant fraction of the thermal energy in the cooling flow, the correlation between radio emission and the presence of cooling flows is probably indicating the existence of interactions between the radio plasma and the centres of such cooling flows. However it is important to note that there is only a weak correlation between the $1.4-\mathrm{GHz}$ radio power $(\mathrm{HBvBM})$ and the 6-cm radio power (Burns 1990) with $\dot{M}$.

(xiii) The CCGs in A 1795 and A 2597 host blue lobes of optical continuum along the edges of their radio lobes (McNamara \& O'Connell 1993; Sarazin et al. 1995; McNamara et al. 1996a,b). Through the analysis of numerical simulations De Young (1995) has shown that the blue continuum can be due to young stars produced by jet-induced star formation. This author has suggested that the most appealing scenario consists of short-lived jets (with ages less than $10^{7} \mathrm{yr}$ ), with a high-pressure cooling flow being responsible for the disruption of the jets.

(xiv) Using an X-ray colour/deprojection technique, Allen \&
Fabian (1997a,b) have proved that all the cooling flow galaxies in their sample exhibit significant central concentrations of cooling gas. These authors find that intrinsic X-ray absorption increases with decreasing radius, and that significant excess absorption is not present in non-cooling flow clusters. The estimated times required for the cooling flows to accumulate the observed absorbing gas are typically of only a few $10^{8} \mathrm{yr}$.

Summarizing, all these results suggest that very likely the presence of cooling flows, emission lines, blue excesses, radio emission, soft X-ray-absorbing gas and star formation are different aspects of the same phenomenon. As we have previously mentioned (Section 5.1, Fig. 8g), McNamara (1997) has suggested that his morphological classification of the blue structure in cooling flow CCGs reflects an evolutionary sequence. This author proposes the following scenario: central cluster galaxies accrete $\sim 10^{8} \mathrm{M}_{\odot}$ of gas from a gas-rich dwarf galaxy or a cooling parcel of hot gas. The accreted gas flows to the galaxy centre where it triggers a radio source (by providing the necessary fuel), which subsequently induces a burst of star formation due to the rapid collapse of cold clouds compressed by shocks along the expanding radio source (type 3 , lobe). In a few $\sim 10^{7} \mathrm{yr}$ the lobes disperse (type 4 , amorphous), leading finally to an unresolved point source (type 1). The type 2 (disc) objects do not fall inside this evolutionary sequence. As McNamara (1997) points out, Hydra A is an example of tidal accretion and likely not accretion from the cooling flow. In this sense, Melnick et al. (1997) have proven that the nuclear emission lines in this galaxy originate in a gaseous disc-like structure with a rotational velocity of $\sim 300 \mathrm{~km} \mathrm{~s}^{-1}$. Under this hypothesis, it is not surprising that the behaviour of the central galaxy of Hydra A in Figs 7, 12, 13 and 14 is different from that of the remaining cooling flow objects. The locus of N 1275 in Figs 7(a) and (c) is also suspicious. However this object exhibits such unusual properties that it has been interpreted either as a system of colliding galaxies (Baade \& Minkowski 1954; Minkowski 1957; Rubin et al. 1977; Hu et al. 1983; Holtzman et al. 1992), two superposed galaxies without interaction (De Young, Roberts \& Saslaw 1973), or even an exploding galaxy (Burbidge, Burbidge \& Sandage 1963; Burbidge \& Burbidge 1965). Thus, it is likely that the morphology displayed by this galaxy is more complicated than that naively expected from the cooling flow phenomenon.

If this scenario is true, an important question is why are we observing so many objects with signatures of recent star formation. Given the relatively large fraction of type 3 objects observed in our sample (at least two: A 1795 and A 2597), and considering the timescale for the transition of a CCG throughout this stage (a few $\sim 10^{7} \mathrm{yr}$ ), the scenario proposed by McNamara (1997) is not tenable unless radio-triggered star formation takes place several times during the lifetime of the central galaxies. Some indication that this could well be the case comes from the study carried out by Allen (1995), who found that the number ratios of stars of different spectral types needed to reproduce the UV/blue continua in a sample of CCGs also favour a scenario in which star formation occurs in a series of bursts. The analysis we have presented here (Section 5 ) also suggests that a young burst ( $\sim 0.1 \mathrm{Gyr}$ age $)$ is able to explain the observed spectral properties of the emission-line CCGs better than more continuous star formation. The population synthesis models, in conjunction with the spectral data, also indicate that the total mass of cooled gas transformed into stars is roughly equal to the mass accreted inside the emission-line-region over a $\sim 10^{8} \mathrm{yr}$ period. Interestingly, the accumulation time-scale derived by Allen \& Fabian (1997a,b) to reproduce their calculated 
masses of absorbing gas is also only a few $10^{8}$ yr. Since the observed blue light would be completely dominated by the stars formed in the last burst, it is possible that a sequence of bursts has occured before the most recent one, fuelled by the accreted gas. Since cooling flows can be disrupted by cluster-cluster interactions (Burns et al. 1997), it is also possible that the sequence of bursts takes place only occasionally. As Crawford (1997) pointed out, if rich clusters were built up hierarchically, the subsequent mergers of the subclusters could have disrupted earlier cooling flows, likely high-redshift radio sources (Bremer, Fabian \& Crawford 1997). Therefore, strong cooling flows could have existed in today's rich clusters only over the last $10^{9} \mathrm{yr}$, allowing for a few bursts of star formation (spaced by a few $10^{8} \mathrm{yr}$ ) having taken place during this period.

This scenario accounts for the fate of the gas accreted within the region where emission lines are observed (i.e. the inner $\sim 20 \mathrm{kpc}$ ): it accumulates over periods of a few $10^{8}$ yr and then most of it (perhaps all) forms stars in bursts. Outside this region, star formation signatures are not observed, perhaps because the radio source is unable to trigger star formation at large distances. What happens to the gas depossited there is not clear: some of it is observed as cool material out to $\simeq 100 \mathrm{kpc}$ (Allen \& Fabian 1997a,b), but our lack of knowledge of the physics of star formation prevents us from understanding why (massive) stars are not formed.

This scenario could also provide a tentative explanation of why there are CCGs in clusters with strong cooling flows that do not show any signs of recent star formation: a powerful radio source is also necessary to trigger or catalyz the formation of (massive) stars. The cooling flow provides the fuel, but without a central engine the radio source, needed to kick-start the star formation, would not develop. The poor correlation between radio power and star formation indicators (e.g., $\mathrm{H} \alpha$ fluxes) could be accounted for by the difference in time-scales involved in the radio phenomenon $\left(\sim 10^{7} \mathrm{yr}\right)$ and the star-formation signatures $\left(\sim 10^{8} \mathrm{yr}\right)$. There could also be some delay between the onset of a powerful radio source and the begining of the star formation, which could explain the few cases where a radio source is present in cooling galaxies without clear signs of excess blue light.

We must stress that this picture, although very suggestive, is highly speculative, and further observational work is clearly needed. Our study of optical absorption features has proven to be an excellent tool to investigate the effect of cooling flows in central dominant galaxies, but we recognise that extending the wavelength baseline of the observations is necessary to provide stronger constraints. Moreover, a systematic study of the spatial extent and morphology of the star formation (e.g. via $\mathrm{H} \alpha$ images) and its correlation with radio emission would provide essential information on the suggested link between radio activity and star formation. Near-infrared images would add to the picture by characterizing the distribution of the underlying old stars.

Progress is also required in the detailed modelling of the behaviour of the spectral features for potentially complex stellar populations with a mixture of ages, metal abundances and element ratios. A missing ingredient is a reliable empirical calibration of some spectral features with intrinsic stellar parameters. At present we are near to completing the $\mathrm{D}_{4000}$ calibration in the Lick stellar library (Cardiel, Gorgas \& Pedraz, in preparation).

\section{ACKNOWLEDGMENTS}

Valuable discussions with Steven Allen, Carolin Crawford, Alastair Edge, Andy Fabian, Roderick Johnstone, Paul Nulsen, Jesús Gallego and Armando Gil de Paz are gratefully acknowledged. We thank Brian McNamara for a careful reading of the manuscript, and Clovis Peres for kindly providing the mass deposition rates and cooling radii employed in this paper prior to their publication. NCL would like to thank the Institute of Astronomy, Cambridge, for its hospitality. The WHT is operated on the island of La Palma by the Royal Greenwich Observatory at the Observatorio del Roque de los Muchachos of the Instituto de Astrofísica de Canarias. The Calar Alto Observatory is operated jointly by the Max-Planck-Institute für Astronomie, Heidelberg, and the Spanish Comisión Nacional de Astronomía. This work was supported in part by the Spanish 'Programa Sectorial de Promoción del Conocimiento' under grant No. PB96-610. AAS acknowledges generous financial support from the Royal Society.

\section{REF EREN C ES}

Allen S. W., 1995, MNRAS, 276, 947

Allen S. W., Fabian A. C., 1997a, in Soker N., ed., ASP Conf. Ser. Vol. 115, Galactic and Cluster Cooling Flows. Astron. Soc. Pac., San Francisco, p. 21

Allen S. W., Fabian A. C., 1997b, MNRAS, 286, 583

Allen S. W. et al., 1992, MNRAS, 259, 67

Allen S. W., Fabian A. C., Johnstone R. M., White D. A., Daines S. J., Edge A. C., Stewart G. C., 1993, MNRAS, 262, 901

Allen S. W., Fabian A. C., Edge A. C., Böhringer H., White D. A., 1995, MNRAS, 275, 741

Antonucci R., Barvainis R., 1994, AJ, 1994, 448

Arnaud K. A., 1988, in Fabian A. C., ed., Proc. NATO Advanced Research Workshop, Cooling Flows in Clusters and Galaxies. Kluwer, Dordrecht, p. 31

Baade W., Minkowski R., 1954, ApJ, 119, 215

Baum S., 1992, in Fabian A. C., ed., Clusters and Superclusters of Galaxies. Kluwer, Dordrecht, p. 171

Bertola F., Gregg M. D., Gunn J. E., Oemler A., 1986, ApJ, 303, 624

Bremer M. N., Fabian A. C., Crawford C. S., 1997, MNRAS, 284, 213

Bruzual A. G., 1983, ApJ, 273, 105

Burbidge M., Burbidge G., 1965, ApJ, 142, 1351

Burbidge M., Burbidge G., Sandage A., 1963, Rev. Mod. Phys., 35, 947

Burns J. O., 1990, AJ, 99, 14

Burns J. O., Loken C., Gómez P., Rizza E., Bliton M., Ledlow M., Owen F. N., 1997, in Soker N., ed., ASP Conf. Ser. Vol. 115, Galactic and Cluster Cooling Flows. Astron. Soc. Pac., San Francisco, p. 21

Calzetti D., 1997, preprint (astro-ph/9706121)

Cardiel N., Gorgas J., Aragón-Salamanca A., 1995, MNRAS, 277, 502 (CGA95)

Cardiel N., Gorgas J., Cenarro J., González J. J., 1998, A\&AS, 127, 597

Carollo C. M., Danziger I. J., 1994, MNRAS, 270, 523

Carollo C. M., Danziger I. J., Buson L., 1993, MNRAS, 265, 553

Crawford C. S., 1997, in Soker N., ed., ASP Conf. Ser. Vol. 115, Galactic and Cluster Cooling Flows. Astron. Soc. Pac., San Francisco, p. 38

Crawford C. S., Fabian A. C., 1993, MNRAS, 265, 431

Crawford C. S., Arnaud K. A., Fabian A. C., Johnstone R. M., 1989, MNRAS, 236, 277

Crawford C. S., Edge A. C., Fabian A. C., Allen S. W., Böhringer H., Ebeling H., McMahon R. G., Voges W., 1995, MNRAS, 274, 75

Daines S. J., Fabian A. C., Thomas P. A., 1994, MNRAS, 268, 1060

David L. P., Jones C., Forman W., 1994, ApJ, 428, 544

Davidge T. J., Clark C. C., 1994, AJ, 107, 946

Davidge T. J., Grinder M., 1995, AJ, 109, 1433

Davies R. L., Sadler E. M., Peletier R. F., 1993, MNRAS, 262, 650

De Young D. S., 1995, ApJ, 446, 521

De Young D. S., Roberts M. S., Saslaw W. C., 1973, ApJ, 185, 809

Donahue M., Voit G. M., 1993, ApJ, 414, L17

Donahue M., Voit G. M., 1997, in Soker N., ed., ASP Conf. Ser. Vol. 115, Galactic and Cluster Cooling Flows. Astron. Soc. Pac., San Francisco p. 48 
Edge A. C., Stewart G. C., Fabian A. C., 1992, MNRAS, 258, 177

Faber S. M., 1977, in Tinsley B. M., Larson R. B., eds, The Evolution of Galaxies and Stellar Populations. Yale University Observatory, New Haven, p. 157

Faber S. M., Friel E. D., Burstein D., Gaskell C. M., 1985, ApJS, 57, 711

Fabian A. C., 1994, ARA\&A, 32, 277

Fabian A. C., Nulsen P. E. J., Canizares C. R., 1984, Nat, 310, 733

Fabian A. C., Nulsen P. E. J., Canizares C. R., 1991, A\&AR, 2, 191

Fabian A. C., Arnaud K. A., Bautz M. W., Tawara Y., 1994a, ApJ, 436, L63

Fabian A. C., Johnstone R. M., Daines S. J., 1994b, MNRAS, 271, 737

Fisher D., Franx M., Illingworth G., 1995, ApJ, 448, 119, (FFI95)

González J. J., 1993, PhD thesis, Univ. California, Santa Cruz

González J. J., Gorgas J., 1995, in Buzzoni A., Renzini A., Serrano A., eds, ASP Conf. Ser. Vol. 86, Fresh Views of Elliptical Galaxies. Astron. Soc. Pac., San Francisco, p. 225

Gorgas J., Efstathiou G., Aragón-Salamanca A., 1990, MNRAS, 245, 217

Gorgas J., Faber S. M., Burstein D., González J. J., Courteau S., Prosser C., 1993, ApJS, 86, 153

Goudfrooij P., Emsellem E., 1996, A\&A, 306, L45

Hansen L., Jorgensen H. E., Norgaard-Nielsen H. U., 1995, A\&A, 297, 13

Heckman T. M., Baum S. A., van Breugel W. J. M., McCarthy P., 1989, ApJ, 338, 48 (HBvBM)

Hoessel J. G., 1980, ApJ, 241, 493

Hoessel J. G., Gunn J. E., Thuan T. X., 1980, ApJ, 241, 486

Holtzman J. A. et al., 1992, AJ, 103, 691

Hu E. M., 1992, ApJ, 391, 608

Hu E. M., Cowie L. L., Kaaret P., Jenkins E. B., York D. G., Roesler F. L., 1983, ApJ, 275, L27

Irwin J. A., Sarazin C. L., 1995, ApJ, 455, 497

Jaffe W., 1992, in Fabian A. C., ed., Clusters and Superclusters of Galaxies. Kluwer, Dordrecht, p. 109

Jaffe W., Bremer M. N., 1997, MNRAS, 284, L1

Johnstone R. M., Fabian A. C., Nulsen P. E. J., 1987, MNRAS, 224, 75 (JFN87)

Johnstone R. M., Fabian A. C., Edge A. C., Thomas P. A., 1992, MNRAS, 255,431

Kimble R. A., Davidsen A. F., Sandage A. R., 1989, Ap\&SS, 157, 237

Laor A., 1997, ApJ, 483, L103

Lucey J. R., Guzmán R., Steel J., Carter D., 1997, MNRAS, 287, 899

Massey P., Strobel K., Barnes J. V., Anderson E., 1988, ApJ, 328, 315

McNamara B. R., 1995, ApJ, 443, 77

McNamara B. R., 1997, in Soker N., ed., ASP Conf. Ser. Vol. 115, Galactic and Cluster Cooling Flows. Astron. Soc. Pac., San Francisco, p. 109

McNamara B. R., Jaffe W., 1994, A\&A, 281, 673

McNamara B. R., O’Connell R. W., 1989, AJ, 98, 2018 (MO89)

McNamara B. R., O’Connell R. W., 1992, ApJ, 393, 579 (MO92)

McNamara B. R., O’Connell R. W., 1993, AJ, 105, 417

McNamara B. R., Bregman J. N., O’Connell R. W., 1990, 360, 20

McNamara B. R., Wise M., Sarazin C. L., Jannuzi B. T., Elston R., 1996a, ApJ, 466, L9

McNamara B. R., Jannuzi B. T., Elston R., Sarazin C. L., Wise M., 1996b, ApJ, 469, 66

Melnick J., Gopal-Krishna, Terlevich R., 1997, A\&A, 318, 337

Minkowski R., 1957, in van der Hulst H. C., ed., IAU Symp. 4, Radio Astronomy. Cambridge University Press, Cambridge, p. 107

Miyaji T. et al., 1993, ApJ, 419, 66

Munn J. A., 1992, ApJ, 399, 444

Nørgaard-Nielsen H. U., Jørgensen H. E., Hansen L., 1990, A\&A, 240, 70

O’Dea C. P., Baum S. A., Maloney P. R., Tacconi L. J., Sparks W. B., 1994, ApJ, 422, 467

O’Dea C. P., Gallimore J. F., Baum S. A., 1995, AJ, 109, 260

Oke J. B., 1990, AJ, 99, 1621

Peres C. B., Fabian A. C., Edge A. C., Allen S. W., Johnstone R. M., White D. A., 1998, preprint

Pinkney J. et al., 1996, ApJ, 468, L13

Romanishin W., 1987, ApJ, 323, L113

Rubin V. C., Ford W. K., Peterson C. J., Oort J. H., 1977, ApJ, 211, 693

Salpeter E. E., 1955, ApJ, 121, 161
Sarazin C. L., 1986, Rev. Mod. Phys., 58, 1

Sarazin C. L., 1997, in Soker N., ed., ASP Conf. Ser. Vol. 115, Galactic and

Cluster Cooling Flows. Astron. Soc. Pac., San Francisco, p. 172

Sarazin C. L., O’Connell R. W., 1983, ApJ, 268, 552

Sarazin C. L., Burns J. O., Roettiger K., McNamara B. R., 1995, ApJ, 447, 559

Savage B. D., Mathis J. S., 1979, ARA\&A, 17, 73

Scalo J. M., 1986, Fund. Cosmic Phys., 11, 1

Shields J. C., Filippenko A. V., 1990, ApJ, 353, L7

Smith E. P., Bohlin R. C., Bothun G. D., O’Connell R. W., Roberts M. S., 1997, ApJ, 478, 516

Thomas P. A., Fabian A. C., Nulsen P. E. J., 1987, MNRAS, 228, 973

Thuan T. X., Puschell J. J., 1989, ApJ, 346, 34

Trager S. C., 1997, PhD thesis, Univ. California, Santa Cruz

Voit G. M., Donahue M., 1995, ApJ, 452, 164

Voit G. M., Donahue M., 1997, ApJ, 486, 242

White D. A., Fabian A. C., Johnstone R. M., Mushotzky R. F., Arnaud K. A., 1991, MNRAS, 252, 72

Worthey G., Faber S. M., González J. J., Burstein D., 1994, ApJS, 94, 687

\section{APPENDIX A: RADIAL PROFILE OF THE STAR FORMATION}

We derive the spatial profile of the density of the mass transformed into stars (Fig. 14) assuming that the changes of slope observed in the $\mathrm{D}_{4000}$ gradients in CCGs with cooling flows and emission lines are due, exclusively, to star formation (see Section 5.2). In order to avoid uncertainties in the absolute values of the total $V$ luminosity of the CCGs in the emissionline regions, we only derive the relative radial variations (normalizing both the density of the mass transformed into stars and the spatial extent of the emission-line region).

The extrapolation of the outer $\mathrm{D}_{4000}$ gradients into the emission-line region allows us to estimate the expected radial behaviour of the $\mathrm{D}_{4000}$ corresponding to the underlying old stellar population. Assuming a particular star formation model (in this case we have chosen a single burst with an age $\simeq 0.1 \mathrm{Gyr}$, with solar metallicity and Scalo IMF), it is possible to derive the $f_{V}$ required to reproduce the observed $\mathrm{D}_{4000}$ decrease (i.e. the difference between the measured and the extrapolated $\mathrm{D}_{4000}$ values) as a function of radius.

If we assume that galaxies exhibit spherical geometry, for any concentric shell equation (2) can be written as

$M_{\mathrm{AP}}(k)=f_{V}(k) L_{V}(k)(M / L)_{\mathrm{AP}}$,

where $k$ is an integer which indicates the number of the shell (numbered from the centre to the outside), $M_{\mathrm{AP}}(k)$ and $f_{V}(k)$ are the total mass transformed into stars and the fraction of $V$ light that comes from the accretion population in the $k$ th-shell (respectively), and $(M / L)_{\mathrm{AP}}$ is the mass-to-light ratio of the accretion population given by the adopted stellar population model. The total $V$ luminosity of the $k$ th-shell (in arbitrary units) is computed from the number of counts in the $V$ wavelength range within the spectrograph slit, $N_{V}(k)$, at a radius $r(k)$ (in arbitrary units) using

$L_{V}(k)=N_{V}(k) r(k)$.

Since the mass-to-light ratio of the accretion population is a constant factor and we are only interested in relative values, we can re-write equation (A1) as

$M_{\mathrm{AP}}(k)=f_{V}(k) L_{V}(k)$,

where both $f_{V}(k)$ and $L_{V}(k)$ can be readily evaluated for each $k$ shell. 


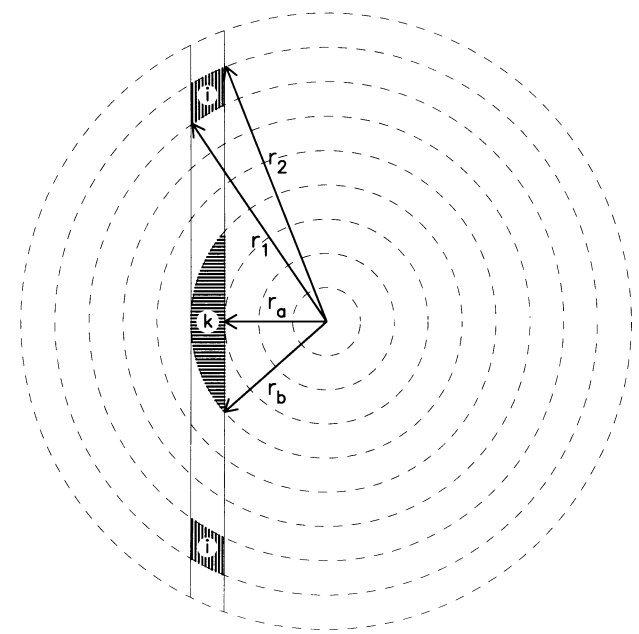

Figure A1. We have obtained the spatial profile of the density of mass transformed into stars $\rho_{\mathrm{AP}}(k)$ in each $k$-shell by subtracting the contribution of the previously determined $\rho_{\mathrm{AP}}(i)\left(k<i \leq N_{\text {shells }}\right)$. For this purpose it is necessary to evaluate the volume of each $i$-shell in the line of sight of the considered $k$-shell, $V(k, i)$. If we define $v\left(r_{\mathrm{c}}, r_{\mathrm{s}}\right)$ as the volume of the intersection between a cylinder of radius $r_{\mathrm{c}}$ and a sphere of radius $r_{\mathrm{s}}$, it is easy to show that $V(k, i)=v\left(r_{\mathrm{b}}, r_{2}\right)-v\left(r_{\mathrm{a}}, r_{2}\right)-v\left(r_{\mathrm{b}}, r_{1}\right)+v\left(r_{\mathrm{a}}, r_{1}\right)$, where $v\left(r_{\mathrm{c}}, r_{\mathrm{s}}\right)=2 \pi r_{\mathrm{c}}^{2} h+\frac{2}{3} \pi\left(r_{\mathrm{s}}-h\right)^{2}\left(2 r_{\mathrm{s}}+h\right)$, where $h^{2}=r_{\mathrm{s}}^{2}-r_{\mathrm{c}}^{2}$.
In order to obtain a realistic three-dimensional radial profile, it is necessary to deproject the measured two-dimensional radial variation. The following deprojection algorithm is based on computation from the outside to the centre. In this sense, the first $M_{\mathrm{AP}}(k)$ computed corresponds to the most external value ( $k=N_{\text {shells }}$, $N_{\text {shells }}$ being the total number of concentric shells), which is not corrected for any projection effect. This initial value is then employed to obtain $\rho_{\mathrm{AP}}\left(N_{\text {shells }}\right)$ by dividing $M_{\mathrm{AP}}\left(N_{\text {shells }}\right)$ by the volume observed in projection in the external shell (in arbitrary units). In successive steps we move towards the central regions (decreasing $k$ ), making use of the already determined $\rho_{\mathrm{AP}}(i)$ values (with $k<i \leq N_{\text {shells }}$ ). In addition

$M_{\mathrm{AP}}(k)=\sum_{i=k}^{N_{\text {shells }}} \rho_{\mathrm{AP}}(i) V(k, i)$,

where $V(k, i)$ is the volume of the $i$ th shell in the line of sight of the $k$ th shell, with $k \leq i \leq N_{\text {shells }}$ (see Fig. A1 and figure caption). Finally, we compute the desired spatial profile employing

$\rho_{\mathrm{AP}}(k)=\frac{1}{V(k, k)}\left[M_{\mathrm{AP}}(k)-\sum_{i=k+1}^{N_{\text {shells }}} \rho_{\mathrm{AP}}(i) V(k, i)\right]$.

This paper has been typeset from a $\mathrm{T}_{\mathrm{E}} \mathrm{X} / \mathrm{L}^{\mathrm{A}} \mathrm{T}_{\mathrm{E}} \mathrm{X}$ file prepared by the author. 